\title{
Shape dependence of entanglement entropy in conformal field theories
}

\section{Thomas Faulkner, Robert G. Leigh and Onkar Parrikar}

Department of Physics, University of Illinois, 1110 W. Green St., Urbana IL 61801-3080, U.S.A.

E-mail: tomf@illinois.edu, rgleigh@illinois.edu, parrika2@illinois.edu

ABSTRACT: We study universal features in the shape dependence of entanglement entropy in the vacuum state of a conformal field theory (CFT) on $\mathbb{R}^{1, d-1}$. We consider the entanglement entropy across a deformed planar or spherical entangling surface in terms of a perturbative expansion in the infinitesimal shape deformation. In particular, we focus on the second order term in this expansion, known as the entanglement density. This quantity is known to be non-positive by the strong-subadditivity property. We show from a purely field theory calculation that the non-local part of the entanglement density in any CFT is universal, and proportional to the coefficient $C_{T}$ appearing in the two-point function of stress tensors in that CFT. As applications of our result, we prove the conjectured universality of the corner term coefficient $\frac{\sigma}{C_{T}}=\frac{\pi^{2}}{24}$ in $d=3$ CFTs, and the holographic Mezei formula for entanglement entropy across deformed spheres.

KEYwords: AdS-CFT Correspondence, Field Theories in Higher Dimensions

ArXiv EPrint: 1511.05179 


\section{Contents}

1 Introduction 1

2 Preliminaries 5

3 The CFT computation $\quad 6$

$\begin{array}{ll}3.1 \text { Relative entropy term } & 10\end{array}$

$\begin{array}{ll}3.2 & \text { The modular Hamiltonian term } \\ & 14\end{array}$

$\begin{array}{lll}3.3 & \text { Spherical case } & 18\end{array}$

4 Applications $\quad 19$

4.1 Corner terms in $d=3 \quad 19$

4.2 Mezei formula 21

5 Discussion $\quad 23$

A Perturbative change in the reduced density matrix $\quad 25$

$\begin{array}{ll}\text { B Angular ordering in the relative entropy term } & 27\end{array}$

C Contact \& crossing terms $\quad 29$

$\begin{array}{lll}\text { C.1 Contact terms } & 29\end{array}$

C.2 Crossing terms 30

$\begin{array}{ll}\text { D Integral } & 31\end{array}$

E Quicker argument for the vanishing of the modular Hamiltonian term 33

\section{Introduction}

Entanglement entropy has a central position in the study of quantum field theories. It is a powerful tool to probe the structure of quantum states, primarily because: (i) it is sufficiently non-local to capture certain global properties, and (ii) it is geometric by definition and hence universal in its applicability. As a result, entanglement entropy has provided great insights in a wide class of systems such as relativistic field theories [1-3], conformal field theories (CFTs) [4-6], topologically ordered phases of matter [7-10], strongly-coupled theories with holographic duals [11-13], etc. It has also become clear that entanglement will play a crucial role in understanding the emergence of geometry in the AdS/CFT correspondence $[14,15]$. Despite this, computing entanglement entropy for arbitrary shaped regions in general dimension still remains a non-trivial task, especially outside the arena 


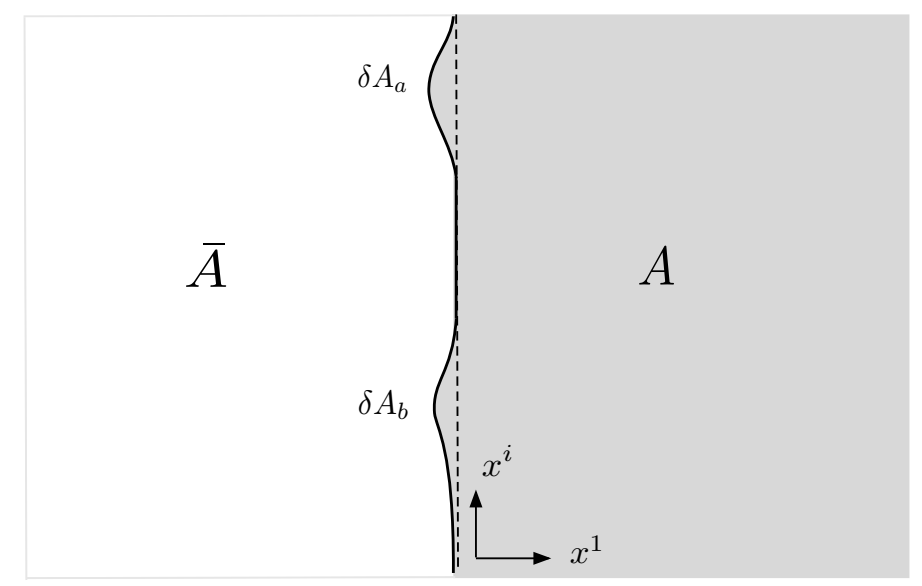

Figure 1. The set-up for the planar case: the original subregion $A$ is the half-space $x^{1}>0$, with the entangling surface $x^{1}=0$ (dashed line). We deform the entangling surface to $x^{1}=-\epsilon \chi\left(x^{i}\right)$ (bold line) by glueing on the area elements $\delta A_{a, b}$ at points $x_{a, b}$ along the entangling surface.

of quantum field theories with classical gravitational duals. While much progress can be made in special symmetric cases such as the entanglement entropy across planar surfaces in relativistic quantum field theories, spherical surfaces in CFTs, etc., it is desirable to develop a larger theoretical toolkit.

In this paper, we study entanglement entropy for deformed half-spaces and ball-shaped regions in the vacuum state of a conformal field theory on $\mathbb{R}^{1, d-1}$. To be concrete, we first explain our construction for deformed half-spaces (see figure 1). Let us pick global coordinates $\boldsymbol{x}^{\mu}=\left(x^{0}, x^{1}, \cdots, x^{d-1}\right)$ on $\mathbb{R}^{1, d-1}$, where $x^{0}$ is the time coordinate. Pick the Cauchy surface $x^{0}=0$, and consider the reduced density matrix $\widehat{\rho}_{0}$ on the half space $A$ given by

$$
A=\left\{\boldsymbol{x}^{\mu} \in \mathbb{R}^{1, d-1} \mid x^{0}=0, x^{1}>0\right\} .
$$

The entanglement entropy between $A$ and its complement $\bar{A}$ is defined as the Von Neumann entropy of $\widehat{\rho}_{0}$. Next, deform the region $A$ slightly to

$$
A+\delta A=\left\{\boldsymbol{x}^{\mu} \in \mathbb{R}^{1, d-1} \mid x^{0}=0, x^{1}>-\epsilon \chi\left(x^{2}, \cdots, x^{d-1}\right)\right\}
$$

where $\chi\left(x^{2}, \cdots, x^{d-1}\right)$ is a smooth function of the $(d-2)$ transverse spatial coordinates (parametrizing the entangling surface) which we denote collectively as $x^{i}=\left(x^{2}, \cdots, x^{d-1}\right)$, and $\epsilon$ is a positive infinitesimal parameter. This corresponds to deforming the entangling surface to $x^{1}=-\epsilon \chi\left(x^{i}\right)$ within the original Cauchy surface. We can also generalize this and deform the entangling surface by the infinitesimal vector field $\zeta^{\bar{i}}\left(x^{i}\right)$, which we take to lie in the plane perpendicular to the original surface $x^{1}=0$ (i.e., the overlined indices run over $\bar{i}=0,1)$, and which also includes, for instance, time-like deformations. The entanglement entropy across the deformed surface can be written as a perturbative expansion in $\zeta^{\bar{i}}$

$$
\begin{aligned}
S_{E E}[A+\delta A]= & S_{E E}[A]+\int d^{d-2} x \zeta^{\bar{i}}(x) S_{\bar{i}}^{(1)}(x) \\
& +\frac{1}{2 !} \int d^{d-2} x_{a} d^{d-2} x_{b} \zeta^{\bar{i}}\left(x_{a}\right) \zeta^{\bar{j}}\left(x_{b}\right) S_{\overline{i j}}^{(2)}\left(x_{a}, x_{b}\right)+\cdots
\end{aligned}
$$


The quantity $S_{\overline{i j}}^{(2)}\left(x_{a}, x_{b}\right)$ is known as the entanglement density $[16-18],{ }^{1}$ and will be the primary focus of the present paper. In the case where $\zeta^{\bar{i}}$ is spacelike and $\zeta^{1}>0$, there is another nice way of thinking about the entanglement density: start with the half space $A$ and glue on to it small area elements $\delta_{a} A^{\bar{i}}$ and $\delta_{b} A^{\bar{i}}$ at the points $x_{a}$ and $x_{b}$ on the entangling surface such that $\delta_{a} A$ and $\delta_{b} A$ are non overlapping. Then to lowest order in $\delta_{a, b} A$, the entanglement density is proportional to the conditional mutual information between $\delta A_{a}$ and $\delta A_{b}$ given the state on $A$

$$
\begin{aligned}
\delta_{a} A^{\bar{i}} \delta_{b} A^{\bar{j}} S_{\overline{i j}}^{(2)}\left(x_{a}, x_{b}\right) & =S_{E E}\left[A+\delta_{a} A+\delta_{b} A\right]-S_{E E}\left[A+\delta_{a} A\right]-S_{E E}\left[A+\delta_{b} A\right]+S_{E E}[A] \\
& =-I\left(A+\delta A_{a} ; \delta A_{b}\right)+I\left(A ; \delta A_{b}\right) \\
& =-I\left(\delta A_{a} ; \delta A_{b} \mid A\right)
\end{aligned}
$$

where $I(X ; Y)$ is the mutual information between the regions $X$ and $Y$. The strong subadditivity property then implies

$$
\delta_{a} A^{\bar{i}} \delta_{b} A^{\bar{j}} S_{\overline{i j}}^{(2)} \leq 0
$$

Consequently, the entanglement density provides a natural notion of a metric on the space of geometries of the entangling surface. In theories with holographic duals, the RyuTakayanagi proposal maps this space into the space of mimimal-area surfaces in the bulk, and so the entanglement density provides a natural metric on the latter space as well (see [20] for more details in the $A d S_{3} / \mathrm{CFT}_{2}$ case). It has also been argued in [18] that in holographic theories, equation (1.5) applied to a special class of deformations maps to the integrated null-energy condition on the bulk minimal-area surface.

In general, entanglement density in conformal field theories can contain two types of terms: (1) contact terms which arise in the coincident limit $x_{a} \rightarrow x_{b}$, and (2) a non-local term which is finite and well-defined when $x_{a}$ and $x_{b}$ are separated. For the most part, we will be interested in the latter. This non-local term is isolated via the definition (1.4) in terms of the conditional mutual information which makes it is clear this term should be independent of the UV cutoff. The main result of the present paper is as follows: for any conformal field theory, the non-local term in the entanglement density for a planar surface is universal and given by

$$
S_{\overline{i j}, \text { non-local }}^{(2)}\left(x_{a}, x_{b}\right)=-\frac{2 \pi^{2} C_{T}}{(d+1)} \frac{\eta_{\overline{i j}}}{\left|x_{a}-x_{b}\right|^{2(d-1)}}
$$

where $C_{T}$ is the numerical coefficient appearing in the two-point function of stress tensors in the CFT. Equation (1.6) was obtained in [17] for a class of holographic theories using the Ryu-Takayanagi formula. However, we emphasize that in this paper we are working with

\footnotetext{
${ }^{1}$ Sometimes entanglement density is defined with an extra minus sign to make it a naturally positive quantity, see (1.5). It is also not clear why one should think of it as a density - entanglement susceptibility would probably be a more appropriate name; however we will follow [16-18] in using the term entanglement density. A similar quantity was studied in [19].
} 


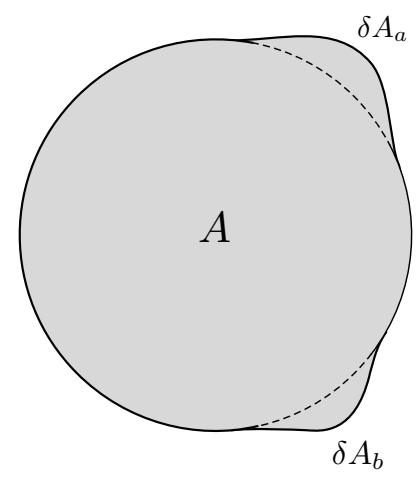

Figure 2. The set-up for the spherical case. We deform the original entangling surface $\mathrm{x}^{2}=R^{2}$ (dashed line) by glueing on the area elements $\delta A_{a, b}$ at points $\Omega_{a, b}$ along the entangling surface.

completely general CFTs. ${ }^{2}$ We will employ purely field theoretic techniques (developed in $[6,21-23]$ ) to prove equation (1.6), thus extending the validity of this formula to arbitrary conformal field theories with or without holographic duals, and further providing a nontrivial check on the Ryu-Takayanagi and the Hubeny-Rangamani-Takayanagi proposals for entanglement entropy in holographic theories.

An analogous formula also holds in the case where we take $A$ to be a ball-shaped region of radius $R$ (see figure 2). Let $\mathbf{x}=\left(x^{1}, \cdots, x^{d-1}\right)$ denote the spatial coordinates on the Cauchy slice $x^{0}=0$ and take $A$ to be the region $\mathbf{x}^{2} \leq R^{2}$. For $\mathbf{x}_{a}=R \Omega_{a}$ and $\mathbf{x}_{b}=R \Omega_{b}$ two well separated points on the entangling surface, the non-local term in the entanglement density is given by

$$
S_{\overline{i j}, \text { non-local }}^{(2)}\left(\Omega_{a}, \Omega_{b}\right)=-\frac{2 \pi^{2} C_{T}}{(d+1) R^{2}} \frac{\eta_{\overline{i j}}}{\left|\Omega_{a}-\Omega_{b}\right|^{2(d-1)}} .
$$

In fact, since a ball-shaped region can be mapped into a half-space by a conformal tranformation, we will argue that equation (1.7) follows as a direct consequence of equation (1.6) in a CFT.

A number of results follow as corollaries: (i) in [24, 25], it was conjectured based on holographic and numerical evidence that the coefficient $a(\theta)$ of the corner term contribution to the entanglement entropy in $d=3$ CFTs has the universal behaviour

$$
\lim _{\theta \rightarrow \pi} a(\theta)=\sigma(\pi-\theta)^{2}+\cdots, \quad \frac{\sigma}{C_{T}}=\frac{\pi^{2}}{24}
$$

where $\theta$ is the opening angle of the corner. We will prove this conjecture as a special case of our results. (ii) We also prove the Mezei formula for the universal part of the entanglement

\footnotetext{
${ }^{2}$ Actually it is enough to invoke the $\mathrm{SO}(1, d-1) \times \mathrm{SO}(1,1)$ conformal symmetries that leave the entangling surface $\partial A$ fixed, including the boosts in the transverse plane, to argue that the cut-off independent part of the entanglement density should take the form as in (1.6). Here we will be tracking down the overall coefficient.
} 
entropy across deformed spheres

$S_{E E}^{(2)}=C_{T} \frac{\pi^{\frac{d+2}{2}}(d-1)}{2^{d-2} \Gamma(d+2) \Gamma(d / 2)} \sum_{\ell, m_{1}, \cdots, m_{d-3}} a_{\ell, m_{1}, \cdots, m_{d-3}}^{2} \prod_{k=1}^{d}(\ell+k-2) \times \begin{cases}(-1)^{\frac{d-1}{2}} \frac{\pi}{2} & d \text { odd } \\ (-1)^{\frac{d-2}{2}} \ln \frac{R}{\delta} & d \text { even }\end{cases}$

which was conjectured in [26] based on holographic calculations in a large class of theories. In (1.9), $a_{\ell, m_{1}, \cdots, m_{d-3}}$ are the coefficients of the expansion of the shape deformation in terms of real hyperspherical harmonics on the entangling surface. The Mezei formula is meant to apply to the universal term in CFT entanglement entropy for a deformed sphere, and the positivity of the overall coefficient demonstrates that the sphere locally minimizes this universal term in the space of shapes, suggesting that the sphere is somehow the optimal measure of degrees of freedom in a CFT for use as an RG monotone. Further, the above formula was used in [27] to compute universal corner contributions to entanglement entropy in higher dimensions. Therefore, our proof of the Mezei formula also completes the proof of universality of corner contributions in higher dimensions. In this way, our CFT calculation fits nicely into the triangle of recent studies and conjectures [17, 23-30] (see also [31-35]) on entanglement density, corner contributions and the Mezei formula.

The rest of the paper is organized as follows: in section 2, we review some elementary facts about entanglement across planar and spherical surfaces, which will be relevant for our subsequent calculations. In section 3, we present the CFT calculation of the universal non-local term in the entanglement density for planar and spherical surfaces. In section 4 , we will then use our result for the entanglement density to prove the universality of corner contributions in $d=3$ CFTs and the Mezei formula. Finally, we will end with some discussion about prospects for future work.

\section{Preliminaries}

Entanglement entropy is defined as follows - consider the density matrix $|\Psi\rangle\langle\Psi|$ corresponding to a pure state defined on the Cauchy surface $\Sigma$. In this paper, we will take $|\Psi\rangle$ to be the ground state of a conformal field theory. Let us partition $\Sigma$ into two subregions $A$ and $\bar{A}$. For local quantum field theories, we expect the Hilbert space $\mathfrak{h}_{\Sigma}$ to factorize into the tensor product $\mathfrak{h}_{\Sigma}=\mathfrak{h}_{A} \otimes \mathfrak{h}_{\bar{A}}$. If this is the case, we can trace over $\mathfrak{h}_{\bar{A}}$ to obtain the reduced density matrix

$$
\widehat{\rho}_{0}=\operatorname{tr}_{\mathfrak{h}_{\bar{A}}}(|\Psi\rangle\langle\Psi|)
$$

which contains all the relevant information pertaining to the subregion $A$. Then the $e n$ tanglement entropy between $A$ and $\bar{A}$ is defined as the von Neumann entropy of $\widehat{\rho}_{0}$

$$
S_{E E}[A]=-\operatorname{tr}_{\mathfrak{h}_{A}}\left(\widehat{\rho}_{0} \ln \widehat{\rho}_{0}\right) .
$$

In this context, the boundary $\partial A$ of $A$ is referred to as the entangling surface. It is also useful to define the modular Hamiltonian (also known as the entanglement Hamiltonian) $\widehat{H}_{E}$ in terms of $\widehat{\rho}_{0}$ as

$$
\widehat{\rho}_{0} \equiv e^{-\widehat{H}_{E}}
$$


In general, the modular Hamiltonian is not a local operator, in the sense that the modular evolution $U(s)=\widehat{\rho}_{0}^{i s}$ does not map local operators to local operators. However, there are a few special cases where symmetry forces the modular Hamiltonian to be local. The simplest such case is when we take $A$ to be the half-space $x^{1}>0$. In this case, the modular Hamiltonian takes a simple form in terms of the CFT defined on Euclidean space $\mathbb{R}^{d}$ :

$$
\widehat{H}_{E, \text { plane }}=2 \pi \widehat{K}+\text { constant }
$$

where $\widehat{K}$ is the generator of rotations around the entangling surface in the $\left(x_{E}^{0}, x^{1}\right)$ plane $\left(x_{E}^{0}\right.$ is Euclidean time)

$$
\widehat{K}=\int d^{d-2} x^{i} \int_{0}^{\infty} d x^{1} x^{1} \widehat{T}^{00}\left(0, x^{1}, x^{i}\right)
$$

and the constant in (2.4) is chosen such that $\operatorname{tr}_{\mathfrak{h}} \widehat{\rho}_{0}=1$. This is known as the BisognanoWichmann theorem [36]. The fact that the modular Hamiltonian for planar entangling surfaces in the vacuum state of a conformal field theory on $\mathbb{R}^{1, d-1}$ is local, and can be written as an integral over the stress tensor will play a crucial role in our calculation of the entanglement density. In fact, the statement of the Bisognano-Wichmann theorem is true for the vacuum state of any relativistic quantum field theory, irrespective of conformal symmetry, and so it should be possible to extend our calculation to the more general class of relativistic quantum field theories. However, in this paper we will restrict ourselves to CFTs, because the calculation simplifies greatly in this case.

In conformal field theories, the modular Hamiltonian for a ball-shaped region (of radius $R)$ is also local [4]. This happens because the conformal transformation

$$
\psi^{\mu}(\boldsymbol{x})=\frac{\boldsymbol{x}^{\mu}-(\boldsymbol{x} \cdot \boldsymbol{x}) C^{\mu}}{1-2(C \cdot \boldsymbol{x})+(\boldsymbol{x} \cdot \boldsymbol{x})(C \cdot C)}+2 R^{2} C^{\mu}
$$

with $C^{\mu}=\left(0, \frac{1}{2 R}, 0, \cdots, 0\right)$, maps the half-space $x^{1}>0$ to the ball-shaped region $\mathbf{x}^{2} \leq R^{2}$. Since conformal transformations are symmetries in a CFT, such a map leaves the ground state invariant, and the reduced density matrix on the ball-shaped region can be related to the reduced density matrix on the half-space by a unitary transformation. Additionally, one can transplant the modular Hamiltonian from the half-space to the ball-shaped region by pushing forward the modular flow by $\psi$, which gives

$$
\widehat{H}_{E, \text { sphere }}=2 \pi \int_{\mathbf{x}^{2} \leq R^{2}} d^{d-1} \mathbf{x} \frac{R^{2}-\mathbf{x}^{2}}{2 R} \widehat{T}^{00}(\mathbf{x})+\text { constant }^{\prime} .
$$

For this reason, the calculation of the entanglement density for ball-shaped regions is no more difficult than the calculation for half-spaces in CFTs.

\section{The CFT computation}

Let us now delve into the calculation of the entanglement density in conformal field theories. For simplicity, we will describe the computation for half spaces in some detail, and then derive the corresponding result for ball-shaped regions by using the conformal transformation mentioned previously. So take $A$ to be the half-space $x^{1}>0$. Consider now the 
entanglement entropy of the deformed region $A+\delta A$ given by $x^{1}>-\epsilon \chi\left(x^{i}\right)$. In order to compute this entropy, we can use the coordinate transformation

$$
\boldsymbol{x}^{\mu} \rightarrow \boldsymbol{x}^{\mu}-\zeta^{\mu}(x), \quad \zeta^{\mu}=-\left(0, \epsilon \chi\left(x^{i}\right), 0, \cdots, 0\right)
$$

to map the deformed entangling region $A+\delta A$ back to the half-space $A$. However, we must bear in mind that such a coordinate transformation has a non-trivial action on the metric. In terms of the new coordinates, the metric is given by

$$
g_{\mu \nu}=\eta_{\mu \nu}+2 \partial_{(\mu} \zeta_{\nu)}+O\left(\epsilon^{2}\right) .
$$

Therefore, to compute the entanglement entropy for $A+\delta A$ in flat space, we may equivalently compute the entanglement entropy for the half-space $A$ but with the deformed metric $g_{\mu \nu}[37]^{3}$

$$
S_{E E}\left[A+\delta A, \eta_{\mu \nu}\right]=S_{E E}\left[A, g_{\mu \nu}\right] .
$$

For our purpose it suffices to keep only the term linear in $\zeta^{\mu}$ in equation (3.2) because we are interested in computing the non-local contribution to the entanglement entropy at second order in the perturbation series, while the $O\left(\epsilon^{2}\right)$ terms in (3.2) can at most generate a local contribution at this order. The shape deformation in (3.1) is somewhat special in that it preserves the Cauchy surface $x^{0}=0$. In our calculation we will relax this and consider the more general deformation

$$
\zeta=\zeta^{\bar{i}}\left(x^{i}\right) \partial_{\bar{i}}=\zeta^{0}\left(x^{i}\right) \partial_{0}+\zeta^{1}\left(x^{i}\right) \partial_{1}
$$

which also includes time-like deformations of the entangling surface, and of which equation (3.1) is a special case. ${ }^{4}$

The advantage of trading the original problem of computing $S_{E E}\left[A+\delta A, \eta_{\mu \nu}\right]$ with that of computing $S_{E E}\left[A, \eta_{\mu \nu}+2 \partial_{(\mu} \zeta_{\nu)}\right]$, is that it is possible to use conformal perturbation theory to write an expansion for the latter in terms of the deformation $\delta g_{\mu \nu}=2 \partial_{(\mu} \zeta_{\nu)}[21-23]$. To see how this works, consider the reduced density matrix $\widehat{\rho}$ on $A$ in the presence of the metric deformation $\delta g_{\mu \nu}$. A straightforward calculation shows (see appendix A for details)

$$
\begin{aligned}
\widehat{\rho}= & \widehat{\rho}_{0}+\frac{1}{2} \int d^{d} \boldsymbol{x} \delta g_{\mu \nu}(\boldsymbol{x}) \widehat{\rho}_{0}\left(\widehat{T}^{\mu \nu}(\boldsymbol{x})-\operatorname{tr}\left(\widehat{\rho}_{0} \widehat{T}^{\mu \nu}(\boldsymbol{x})\right)\right) \\
& +\frac{1}{8} \int d^{d} \boldsymbol{x}_{a} d^{d} \boldsymbol{x}_{b} \delta g_{\mu \nu}\left(\boldsymbol{x}_{a}\right) \delta g_{\lambda \sigma}\left(\boldsymbol{x}_{b}\right) \widehat{\rho}_{0}\left\{\mathcal{T}\left[\widehat{T}^{\mu \nu}\left(\boldsymbol{x}_{a}\right) \widehat{T}^{\lambda \sigma}\left(\boldsymbol{x}_{b}\right)\right]-2 \widehat{T}^{\mu \nu}\left(\boldsymbol{x}_{a}\right) \operatorname{tr}\left(\widehat{\rho}_{0} \widehat{T}^{\lambda \sigma}\left(\boldsymbol{x}_{b}\right)\right)\right. \\
& \left.-\operatorname{tr}\left(\widehat{\rho}_{0} \mathcal{T}\left[\widehat{T}^{\mu \nu}\left(\boldsymbol{x}_{a}\right) \widehat{T}^{\lambda \sigma}\left(\boldsymbol{x}_{b}\right)\right]\right)+2 \operatorname{tr}\left(\widehat{\rho}_{0} \widehat{T}^{\mu \nu}\left(\boldsymbol{x}_{a}\right)\right) \operatorname{tr}\left(\widehat{\rho}_{0} \widehat{T}^{\lambda \sigma}\left(\boldsymbol{x}_{b}\right)\right)\right\}+\cdots
\end{aligned}
$$

where $\boldsymbol{x}^{\mu}=\left(x_{E}^{0}, \mathbf{x}\right)$ are now coordinates on Euclidean space $\mathbb{R}^{d}$, and

$$
\widehat{\rho}_{0}=\frac{e^{-2 \pi \widehat{K}}}{\operatorname{tr} e^{-2 \pi \widehat{K}}}
$$

\footnotetext{
${ }^{3}$ Note that (3.3) is true (even for the UV divergent terms) if we use a "covariant" regulator to define EE [38-40]. However since we are ultimately interested in a UV finite quantity the regulator used at intermediate stages in the calculation should not matter.

${ }^{4}$ We need not include components along the transverse directions $\partial_{i}$ because these simply amount to reparametrizations of the entangling surface, which do not change the entanglement entropy.
} 
is the original reduced density matrix on $A$ in the absence of the metric perturbation. ${ }^{5}$ Further, $\mathcal{T}$ is the angular-ordering operator in the $\left(x_{E}^{0}, x^{1}\right)$ plane, i.e., if $\theta \in[0,2 \pi)$ is the angular coordinate in the $\left(x_{E}^{0}, x^{1}\right)$ plane, then

$$
\mathcal{T}\left[\widehat{\mathcal{O}}\left(\theta_{a}\right) \widehat{\mathcal{O}}\left(\theta_{b}\right)\right]=\widehat{\mathcal{O}}\left(\theta_{a}\right) \widehat{\mathcal{O}}\left(\theta_{b}\right) H\left(\theta_{a}-\theta_{b}\right)+\widehat{\mathcal{O}}\left(\theta_{b}\right) \widehat{\mathcal{O}}\left(\theta_{a}\right) H\left(\theta_{b}-\theta_{a}\right)
$$

where $H\left(\theta_{a}-\theta_{b}\right)$ is the Heaviside step function. The next step is to perturbatively expand the entanglement entropy $S_{E E}=-\operatorname{tr}(\widehat{\rho} \ln \widehat{\rho})$ using eqaution (3.5). However, care must be taken in expanding the logarithm, because $\widehat{\rho}_{0}$ and $\delta \widehat{\rho}=\widehat{\rho}-\widehat{\rho}_{0}$ do not commute in general. In order to deal with this, we use the following integral representation for the entanglement entropy

$$
S_{E E}=-\operatorname{tr} \widehat{\rho} \ln \widehat{\rho}=\int_{0}^{\infty} d \beta\left\{\operatorname{tr}\left(\frac{\widehat{\rho}}{\widehat{\rho}+\beta}\right)-\frac{1}{1+\beta}\right\} .
$$

Expanding this out to second order in $\delta \widehat{\rho}$, we obtain

$$
\delta S_{E E}=\int_{0}^{\infty} d \beta \operatorname{tr}\left(\delta \widehat{\rho} \frac{\beta}{\left(\widehat{\rho}_{0}+\beta\right)^{2}}\right)-\int_{0}^{\infty} d \beta \operatorname{tr}\left(\frac{\beta}{\left(\widehat{\rho}_{0}+\beta\right)^{2}} \delta \widehat{\rho} \frac{1}{\left(\widehat{\rho}_{0}+\beta\right)} \delta \widehat{\rho}\right)+\cdots .
$$

Substituting equation (3.5) in (3.9), we find that $\delta S_{E E}$ can be written as a sum of two terms

$$
\delta S_{E E}=\delta S_{E E}^{(1)}+\delta S_{E E}^{(2)}
$$

coming respectively from the first and the second term in equation (3.9). The first term (after performing the $\beta$ integration) is given by

$$
\begin{aligned}
\delta S_{E E}^{(1)}= & \frac{1}{2} \int d^{d} \boldsymbol{x} \delta g_{\mu \nu}(\boldsymbol{x}) \operatorname{tr}_{\text {conn. }}\left(\widehat{\rho}_{0} \widehat{T}^{\mu \nu}(\boldsymbol{x}) \widehat{H}_{E}\right) \\
& +\frac{1}{8} \int d^{d} \boldsymbol{x}_{a} d^{d} \boldsymbol{x}_{b} \delta g_{\mu \nu}\left(\boldsymbol{x}_{a}\right) \delta g_{\lambda \sigma}\left(\boldsymbol{x}_{b}\right) \operatorname{tr} \text { conn. }\left(\widehat{\rho}_{0} \mathcal{T}\left[\widehat{T}^{\mu \nu}\left(\boldsymbol{x}_{a}\right) \widehat{T}^{\lambda \sigma}\left(\boldsymbol{x}_{b}\right)\right] \widehat{H}_{E}\right)
\end{aligned}
$$

where $\widehat{H}_{E}$ is the modular Hamiltonian corresponding to $\widehat{\rho}_{0}$ and $\operatorname{tr}_{\text {conn. }}$ is the connected trace. From the above equation, we see that $\delta S_{E E}^{(1)}$ can be interpreted as the change in the expectation value of the (original) modular Hamiltonian; we will henceforth refer to this term as the modular Hamiltonian term. Given that all the operators inside the trace are naturally $\mathcal{T}$-ordered, we can rewrite the above traces in terms of connected Euclidean correlation functions

$$
\begin{aligned}
\delta S_{E E}^{(1)}= & \frac{1}{2} \int d^{d} \boldsymbol{x} \delta g_{\mu \nu}(\boldsymbol{x})\left\langle\widehat{T}^{\mu \nu}(\boldsymbol{x}) \widehat{H}_{E}\right\rangle_{\mathrm{conn} .} \\
& +\frac{1}{8} \int d^{d} \boldsymbol{x}_{a} d^{d} \boldsymbol{x}_{b} \delta g_{\mu \nu}\left(\boldsymbol{x}_{a}\right) \delta g_{\lambda \sigma}\left(\boldsymbol{x}_{b}\right)\left\langle\widehat{T}^{\mu \nu}\left(\boldsymbol{x}_{a}\right) \widehat{T}^{\lambda \sigma}\left(\boldsymbol{x}_{b}\right) \widehat{H}_{E}\right\rangle_{\text {conn. }} .
\end{aligned}
$$

Now, the second term in (3.10) is given by

$$
\begin{aligned}
\delta S_{E E}^{(2)}= & -\frac{1}{4} \int d^{d} \boldsymbol{x}_{a} d^{d} \boldsymbol{x}_{b} \delta g_{\mu \nu}\left(\boldsymbol{x}_{a}\right) \delta g_{\lambda \sigma}\left(\boldsymbol{x}_{b}\right) \int_{0}^{\infty} d \beta \beta \\
& \times \operatorname{tr}\left\{\frac{\widehat{\rho}_{0}}{\left(\widehat{\rho}_{0}+\beta\right)^{2}}\left[\widehat{T}^{\mu \nu}\left(\boldsymbol{x}_{a}\right)-\operatorname{tr}\left(\widehat{\rho}_{0} \widehat{T}^{\mu \nu}\left(\boldsymbol{x}_{a}\right)\right)\right] \frac{\widehat{\rho}_{0}}{\widehat{\rho}_{0}+\beta}\left[\widehat{T}^{\lambda \sigma}\left(\boldsymbol{x}_{b}\right)-\operatorname{tr}\left(\widehat{\rho}_{0} \widehat{T}^{\lambda \sigma}\left(\boldsymbol{x}_{b}\right)\right)\right]\right\} .
\end{aligned}
$$

\footnotetext{
${ }^{5}$ From now on, by $\operatorname{tr}$ we mean $\operatorname{tr}_{\mathfrak{h}_{A}}$ unless otherwise specified.
} 
This term is in fact the negative of the relative entropy of $\widehat{\rho}$ with respect to $\widehat{\rho}_{0}$ at second order in $\delta g_{\mu \nu}$

$$
\delta S_{E E}^{(2)}=-S\left(\widehat{\rho} \| \widehat{\rho}_{0}\right)=\operatorname{tr}\left(\widehat{\rho} \ln \widehat{\rho}_{0}\right)-\operatorname{tr}(\widehat{\rho} \ln \widehat{\rho})
$$

and will henceforth be referred to as the relative entropy term. The non-negativity of relative entropy then implies

$$
\delta S_{E E}^{(2)} \leq 0
$$

Unfortunately, the operators appearing in equation (3.13) are not manifestly $\mathcal{T}$-ordered, and so the trace in this form cannot be written as a Euclidean correlation function. However, it is possible to perform the $\beta$ integral and manipulate this expression further to bring it to a more convenient form (see appendix B)

$$
\begin{aligned}
\delta S_{E E}^{(2)}= & \frac{1}{32} \int d^{d} \boldsymbol{x}_{a} d^{d} \boldsymbol{x}_{b} \delta g_{\mu \nu}\left(\boldsymbol{x}_{a}\right) \delta g_{\lambda \sigma}\left(\boldsymbol{x}_{b}\right) \int_{-\infty}^{\infty} \frac{d s}{\sinh ^{2}\left(s / 2+i \varepsilon \operatorname{sgn}\left(\theta_{a}-\theta_{b}\right)\right)}\left(R^{-1}(i s)\right)^{\lambda} \\
& \times\left(R^{-1}(i s)\right)^{\sigma}{ }_{\eta} \operatorname{tr}_{\text {conn. }}\left(\widehat{\rho}_{0} \mathcal{T}\left[\widehat{T}^{\mu \nu}\left(\boldsymbol{x}_{a}\right) \widehat{T}^{\kappa \eta}\left(R(i s) \cdot \boldsymbol{x}_{b}\right)\right]\right)
\end{aligned}
$$

where $(R(\theta))_{\nu}^{\mu}$ is a rotation in the $\left(x_{E}^{0}, x^{1}\right)$ plane by the angle $\theta$. This manipulation essentially involves steps similar to passing from old-fashioned perturbation theory to time dependent perturbation theory in quantum mechanics. This is usually achieved using Schwinger parameters and the $s$ appearing above can be thought of as such.

The trade-off however is the additional $s$ integral with the attendant measure. Interestingly, note that the way $s$ appears in the above correlation function corresponds to a relative boost between the two stress tensor insertions, with $s$ being the boost angle/rapidity. Equivalently, from the point of view of the modular Hamiltonian, we are forced into "real time" evolution. Indeed, we can rewrite the above equation in the following way to make this point manifest

$$
\begin{aligned}
\delta S_{E E}^{(2)}= & \frac{1}{32} \int d^{d} \boldsymbol{x}_{a} d^{d} \boldsymbol{x}_{b} \delta g_{\mu \nu}\left(\boldsymbol{x}_{a}\right) \delta g_{\lambda \sigma}\left(\boldsymbol{x}_{b}\right) \\
& \times \int_{-\infty}^{\infty} \frac{d s}{\sinh ^{2}\left(s / 2+i \varepsilon \operatorname{sgn}\left(\theta_{a}-\theta_{b}\right)\right)} \operatorname{tr}_{\text {conn. }}\left(\widehat{\rho}_{0} \mathcal{T}\left[\widehat{T}^{\mu \nu}\left(\boldsymbol{x}_{a}\right) \widehat{\rho}_{0}^{-i s / 2 \pi} \widehat{T}^{\lambda \sigma}\left(\boldsymbol{x}_{b}\right) \widehat{\rho}_{0}^{i s / 2 \pi}\right]\right) .
\end{aligned}
$$

Having written this term in the above form we can now use the $\mathcal{T}$-ordering to rewrite the trace in terms of the Euclidean two-point correlation function to obtain

$$
\begin{aligned}
\delta S_{E E}^{(2)}= & \frac{1}{32} \int d^{d} \boldsymbol{x}_{a} d^{d} \boldsymbol{x}_{b} \delta g_{\mu \nu}\left(\boldsymbol{x}_{a}\right) \delta g_{\lambda \sigma}\left(\boldsymbol{x}_{b}\right) \int_{-\infty}^{\infty} \frac{d s}{\sinh ^{2}\left(s / 2+i \varepsilon \operatorname{sgn}\left(\theta_{a}-\theta_{b}\right)\right)}\left(R^{-1}(i s)\right)_{\kappa}^{\lambda} \\
& \times\left(R^{-1}(i s)\right)_{\eta}^{\sigma}\left\langle\widehat{T}^{\mu \nu}\left(\boldsymbol{x}_{a}\right) \widehat{T}^{\kappa \eta}\left(R(i s) \cdot \boldsymbol{x}_{b}\right)\right\rangle_{\text {conn. }} .
\end{aligned}
$$

From equations (3.12) and (3.18) we see that the entanglement density can be computed in terms of the two-point and three-point Euclidean correlation functions of the stress tensor. Indeed, in any conformal field theory, these correlators are universal and fixed by conformal invariance modulo finitely many parameters [41]. The two-point function in 
particular takes the form

$$
\begin{aligned}
\left\langle\widehat{T}_{\mu \nu}(\boldsymbol{x}) \widehat{T}_{\lambda \sigma}(0)\right\rangle_{\text {conn. }} & =\frac{C_{T}}{|\boldsymbol{x}|^{2 d}}\left(\frac{1}{2} I_{\mu \lambda} I_{\nu \sigma}+\frac{1}{2} I_{\mu \sigma} I_{\nu \lambda}-\frac{1}{d} \delta_{\mu \nu} \delta_{\lambda \sigma}\right) \\
I_{\mu \nu} & =\delta_{\mu \nu}-2 \frac{\boldsymbol{x}_{\mu} \boldsymbol{x}_{\nu}}{\boldsymbol{x}^{2}}
\end{aligned}
$$

and is determined entirely by specifying the single parameter $C_{T}$. The three-point function is more complicated, and in general dimension depends on three independent parameters. Nevertheless, it is clear from the above discussion that the (non-local part of the) entanglement density in a CFT is uniquely determined in terms of the parameters appearing in the two- and three-point correlators.

All that remains now is to explicitly evaluate the integrals in equations (3.12) and (3.18). Doing so, one encounters the following surprising result - the modular Hamiltonian term (3.12) does not contribute to the non-local part of the entanglement density. Since the explicit computation is somewhat tedious, we will defer the details to section 3.2. We also give a quicker more sketchy proof of the vanishing of the modular Hamiltonian term, using a slightly different setup, in appendix E. The non-trivial contribution to the entanglement density then comes entirely from the relative entropy term. Indeed, this is why the result (1.6) for the non-local part of the entanglement density depends only on the single parameter $C_{T}$. We now proceed to compute the relative entropy term.

\subsection{Relative entropy term}

In order to compute the integrals in (3.18), it is much more efficient to use the conformal transformation from $\mathcal{H}=S^{1} \times \mathbb{H}^{d-1}$ to $\mathbb{R}^{d}$ to pull-back and evaluate the integrals on $\mathcal{H}$. To see how this works, let us coordinatize $S^{1} \times \mathbb{H}^{d-1}$ by $y^{\alpha}=\left(\tau, z, x^{i}\right)$, where $\tau$ is periodic with period $2 \pi$, and $\left(z, x^{i}\right)$ are Poincaré coordinates on the hyperbolic space $\mathbb{H}^{d-1}$. The metric on $\mathcal{H}$ in these coordinates is given by

$$
g_{\mathcal{H}}=d \tau^{2}+\frac{d z^{2}+\delta_{i j} d x^{i} d x^{j}}{z^{2}}
$$

The map $\varphi: \mathcal{H} \rightarrow \mathbb{R}^{d}$ given by

$$
\varphi\left(\tau, z, x^{i}\right)=\left(z \sin \tau, z \cos \tau, x^{i}\right)
$$

is a conformal transformation, i.e.

$$
\varphi_{*} g_{\mathbb{R}^{d}}=\Omega^{2}(y) g_{\mathcal{H}}
$$

with $\Omega(y)=z$ being the Weyl factor (and $\varphi_{*}$ being the pullback). This implies that the stress tensors on the two spaces are related by

$$
\widehat{T}^{\mu \nu}(\boldsymbol{x})=\frac{\partial \boldsymbol{x}^{\mu}}{\partial y^{\alpha}} \frac{\partial \boldsymbol{x}^{\nu}}{\partial y^{\beta}} \widehat{T}^{\alpha \beta}(y) \Omega^{-2-d}(y)+\mathcal{S}^{\mu \nu}
$$


where $\mathcal{S}^{\mu \nu}$ denotes additional Schwarzian derivative-type terms, which vanish in odd dimensions, but are present in even dimensions. The integral (3.18) then pulls back to

$$
\delta S_{E E}^{(2)}=\frac{1}{32} \int d \mu_{a} d \mu_{b} h_{\alpha \beta}\left(y_{a}\right) \Omega^{-2}\left(y_{a}\right) h_{\gamma \delta}\left(y_{b}\right) \Omega^{-2}\left(y_{b}\right) \Pi^{\alpha \beta \gamma \delta}\left(y_{a}, y_{b}\right)
$$

where $\Pi^{\alpha \beta \gamma \delta}\left(y_{a}, y_{b}\right)=\int_{-\infty}^{\infty} \frac{d s}{\sinh ^{2}\left(s / 2+i \varepsilon \operatorname{sgn}\left(\tau_{a}-\tau_{b}\right)\right)}\left\langle\widehat{T}^{\alpha \beta}\left(y_{a}\right) \widehat{T}^{\gamma \delta}\left(y_{b}^{s}\right)\right\rangle_{\mathcal{H}}$

where we have defined $y_{b}^{s}=\left(\tau_{b}+i s, z_{b}, x_{b}^{i}\right)$, and $d \mu=\sqrt{\operatorname{det} g_{\mathcal{H}}(y)} d^{d} y$ is the measure on $\mathcal{H}$. Further

$$
h_{\alpha \beta} \Omega^{-2}=2 \nabla_{(\alpha} \xi_{\beta)}+g_{\alpha \beta}^{\mathcal{H}} \xi^{\gamma} \partial_{\gamma} \ln \left(\Omega^{2}\right)
$$

where the vector field $\xi^{\alpha}$ on $\mathcal{H}$ is the push-forward of the vector field $\zeta^{\mu}$ on $\mathbb{R}^{d}$ by $\varphi^{-1}$

$$
\xi=\left(\zeta^{1}\left(x^{i}\right) \cos (\tau)+\zeta^{0}\left(x^{i}\right) \sin (\tau)\right) \partial_{z}+\frac{1}{z}\left(-\zeta^{1}\left(x^{i}\right) \sin (\tau)+\zeta^{0}\left(x^{i}\right) \cos (\tau)\right) \partial_{\tau} .
$$

Note that the Schwarzian terms $\mathcal{S}^{\mu \nu}$ have dropped out of equation (3.25) because of the connectedness of the correlation function. Additionally, we note that the second term in (3.27) can also be dropped by the tracelessness of the stress tensor (more precisely, the trace Ward identity) in conformal field theories. ${ }^{6}$ So we obtain

$$
\delta S_{E E}^{(2)}=\frac{1}{8} \int_{\mathcal{H}} d \mu_{a} \int_{\mathcal{H}} d \mu_{b} \nabla_{(\alpha} \xi_{\beta)}\left(y_{a}\right) \nabla_{(\gamma} \xi_{\delta)}\left(y_{b}\right) \Pi^{\alpha \beta \gamma \delta}\left(y_{a}, y_{b}\right) .
$$

Since the above integrals include integration over hyperbolic space, there are potential divergences coming from the conformal boundary of hyperbolic space at $z=0$. These divergences in the entanglement entropy of course correspond to the short-range entanglement coming from the region close to the entangling surface. One way to regulate such potential divergences is to put a cut-off at $z=\frac{1}{\Lambda}$ (which corresponds to cutting out a tubular neighbourhood around the entangling surface in the original description on Euclidean space). We denote the resulting regulated space as $\mathcal{H}_{\Lambda}$, and rewrite the above integral as

$$
\delta S_{E E}^{(2)}=\frac{1}{8} \int_{\mathcal{H}_{\Lambda}} d \mu_{a} \int_{\mathcal{H}_{\Lambda}} d \mu_{b} \nabla_{(\alpha} \xi_{\beta)}\left(y_{a}\right) \nabla_{(\gamma} \xi_{\delta)}\left(y_{b}\right) \Pi^{\alpha \beta \gamma \delta}\left(y_{a}, y_{b}\right)
$$

Next, integrating by parts and using the diffeomorphism Ward identity, ${ }^{7}$ we arrive at

$$
\delta S_{E E}^{(2)}=\frac{1}{8} \int_{\partial \mathcal{H}_{\Lambda}} d \bar{\mu}_{a} n^{\alpha}\left(y_{a}\right) \xi^{\beta}\left(y_{a}\right) \int_{\partial \mathcal{H}_{\Lambda}} d \overline{\mu_{b}} n^{\gamma}\left(y_{b}\right) \xi^{\delta}\left(y_{b}\right) \Pi_{\alpha \beta \gamma \delta}\left(y_{a}, y_{b}\right)
$$

where $\partial \mathcal{H}_{\Lambda}$ is the boundary of the regulated space $\mathcal{H}_{\Lambda}$ at $z=\frac{1}{\Lambda}, n=\frac{1}{\Lambda} \partial_{z}$ is the outward pointing unit-normal on the boundary, and $d \bar{\mu}$ is the measure induced on the boundary

$$
d \bar{\mu} n^{\alpha}(y)=d \tau d^{d-2} x^{i} \sqrt{\operatorname{det} \gamma_{\partial \mathcal{H}_{\Lambda}}} n^{\alpha}(y)=d \tau d^{d-2} x^{i} \Lambda^{(d-2)} \frac{\delta_{z}^{\alpha}}{\Lambda} .
$$

\footnotetext{
${ }^{6}$ In even dimensions, there are contributions coming from the trace anomaly. However, these contributions are local at the present order. Since we are interested in the non-local part of the entanglement density, we can drop these terms.

${ }^{7}$ Which says that $\nabla_{\alpha}^{a} \Pi^{\alpha \beta \gamma \delta}\left(y_{a}, y_{b}\right)=0$ and $\nabla_{\gamma}^{b} \Pi^{\alpha \beta \gamma \delta}\left(y_{a}, y_{b}\right)=0$ for separated points.
} 
The next thing to compute is the two-point function of stress tensors on $\mathcal{H}$. This can be done efficiently using the embedding space formalism developed in [42]. In this formalism (see section 2 of [6] for a review relevant for this calculation), one considers the larger embedding space (or ambient space) $\mathbb{R}^{1, d+1}$ on which the (Euclidean) conformal group acts linearly. Let us pick global coordinates $P^{A}=\left(P^{I}, P^{I I}, P^{0}, \cdots, P^{d-1}\right)$ on this space, with the coordinate $P^{I}$ being time-like. One then embeds $\mathcal{H}$ (more generally, any space which is conformally equivalent to $\mathbb{R}^{d}$ ) as a section of the upper light-cone $P^{2}=0, P^{I}>0$. Here, we pick the embedding

$$
P^{A}(y)=\left(\frac{1+z^{2}+\delta_{i j} x^{i} x^{j}}{2 z}, \cos (\tau), \sin (\tau), \frac{1-z^{2}-\delta_{i j} x^{i} x^{j}}{2 z}, \frac{x^{i}}{z}\right) .
$$

Now the two-point function of stress tensors in equation (3.26) can be computed using the embedding space formalism following [42],

$$
\left\langle\widehat{T}_{\alpha \beta}\left(y_{a}\right) \widehat{T}_{\gamma \delta}\left(y_{b}^{s}\right)\right\rangle_{\mathcal{H}_{\Lambda}}=\frac{1}{4} \mathbb{P}_{\alpha \beta}^{A B}\left(P_{a}\right) \mathbb{P}_{\gamma \delta}^{C D}\left(P_{b}^{s}\right) \frac{\partial}{\partial Z_{a}^{A}} \frac{\partial}{\partial Z_{a}^{B}} \frac{\partial}{\partial Z_{b}^{C}} \frac{\partial}{\partial Z_{b}^{D}} G^{(2)}\left(P_{a}, P_{b}^{s}, Z_{a}, Z_{b}\right)
$$

where $Z_{a, b}$ are auxiliary variables, and the right hand side above is evaluated on the section (3.33). The index-free function $G^{(2)}$ is defined as,

$$
G^{(2)}\left(P_{a}, P_{b}^{s}, Z_{a}, Z_{b}\right)=\frac{4 C_{T}}{\left(-2 P_{a} \cdot P_{b}^{s}\right)^{d+2}}\left(\left(P_{a} \cdot P_{b}^{s}\right)\left(Z_{a} \cdot Z_{b}\right)-\left(P_{a} \cdot Z_{b}\right)\left(P_{b}^{s} \cdot Z_{a}\right)\right)^{2} .
$$

Further, the projector $\mathbb{P}_{\alpha \beta}^{A B}$ is defined as (see equations (3.66) and (3.67) for explicit expressions)

$$
\mathbb{P}_{\alpha \beta}^{A B}(P)=\frac{\partial P^{(A}}{\partial y^{\alpha}} \frac{\partial P^{B)}}{\partial y^{\beta}}-\frac{1}{d} \eta^{A B} \eta_{C D} \frac{\partial P^{C}}{\partial y^{\alpha}} \frac{\partial P^{D}}{\partial y^{\beta}} .
$$

Using this formalism, we compute the required two-point correlation functions

$$
\begin{aligned}
\left\langle\widehat{T}_{z \tau}\left(y_{a}\right) \widehat{T}_{z \tau}\left(y_{b}^{s}\right)\right\rangle_{\mathcal{H}_{\Lambda}} & =\frac{C_{T}\left|x_{a}-x_{b}\right|^{4}\left(\cos \left(2\left(\tau_{a}-\tau_{b}-i s\right)\right)+O\left(\Lambda^{-2}\right)\right)}{2 \Lambda^{2 d-2}\left(\left|x_{a}-x_{b}\right|^{2}+\frac{2}{\Lambda^{2}}\left(1-\cos \left(\tau_{a}-\tau_{b}-i s\right)\right)\right)^{d+2}} \\
\left\langle\widehat{T}_{z \tau}\left(y_{a}\right) \widehat{T}_{z z}\left(y_{b}^{s}\right)\right\rangle_{\mathcal{H}_{\Lambda}} & =-\frac{C_{T}\left|x_{a}-x_{b}\right|^{4}\left(\sin \left(2\left(\tau_{a}-\tau_{b}-i s\right)\right)+O\left(\Lambda^{-2}\right)\right)}{2 \Lambda^{2 d-3}\left(\left|x_{a}-x_{b}\right|^{2}+\frac{2}{\Lambda^{2}}\left(1-\cos \left(\tau_{a}-\tau_{b}-i s\right)\right)\right)^{d+2}} \\
\left\langle\widehat{T}_{z z}\left(y_{a}\right) \widehat{T}_{z z}\left(y_{b}^{s}\right)\right\rangle_{\mathcal{H}_{\Lambda}} & =\frac{C_{T}\left|x_{a}-x_{b}\right|^{4}\left(\cos \left(2\left(\tau_{a}-\tau_{b}-i s\right)\right)+\frac{d-2}{d}+O\left(\Lambda^{-2}\right)\right)}{2 \Lambda^{2 d-4}\left(\left|x_{a}-x_{b}\right|^{2}+\frac{2}{\Lambda^{2}}\left(1-\cos \left(\tau_{a}-\tau_{b}-i s\right)\right)\right)^{d+2}}
\end{aligned}
$$

where $\left|x_{a}-x_{b}\right|^{2}=\delta_{i j}\left(x_{a}-x_{b}\right)^{i}\left(x_{a}-x_{b}\right)^{j}$. The $O\left(\Lambda^{-2}\right)$ terms in the above expressions do not contribute in the limit $\Lambda \rightarrow \infty$, so we will drop them henceforth. Substituting equations (3.37)-(3.39) in (3.31) and using (3.26), we obtain

$$
\delta S_{E E}^{(2)}=\frac{C_{T}}{16 \Lambda^{2}} \int_{0}^{2 \pi} d \tau_{a} \int d^{d-2} x_{a} \int_{0}^{2 \pi} d \tau_{b} \int d^{d-2} x_{b}\left|x_{a}-x_{b}\right|^{4} \hat{\xi}_{a}^{\bar{i}} \mathcal{M}_{\overline{i j}} \hat{\xi}_{b}^{\bar{j}}
$$


where the overlined indices run over $\bar{i}, \bar{j}=(\tau, z)$, and

$$
\hat{\xi}=\left(\zeta^{1} \cos (\tau)+\zeta^{0} \sin (\tau)\right) \partial_{z}+\left(-\zeta^{1} \sin (\tau)+\zeta^{0} \cos (\tau)\right) \partial_{\tau}
$$

We have also defined

$$
\mathcal{M}_{\overline{i j}}=\int_{-\infty}^{\infty} \frac{d s}{\sinh ^{2}\left(s / 2+i \varepsilon \operatorname{sgn}\left(\tau_{a}-\tau_{b}\right)\right)} \frac{1}{\left(\left|x_{a}-x_{b}\right|^{2}+\frac{2}{\Lambda^{2}}\left(1-\cos \left(\tau_{a}-\tau_{b}-i s\right)\right)\right)^{d+2}} M_{\overline{i j}}
$$

with $M_{\overline{i j}}$ given by the two dimensional matrix

$$
M_{\overline{i j}}=\left(\begin{array}{ll}
\cos \left(2\left(\tau_{a}-\tau_{b}-i s\right)\right) & -\sin \left(2\left(\tau_{a}-\tau_{b}-i s\right)\right) \\
\sin \left(2\left(\tau_{a}-\tau_{b}-i s\right)\right) & \cos \left(2\left(\tau_{a}-\tau_{b}-i s\right)\right)+\frac{d-2}{d}
\end{array}\right) .
$$

The factor of $\frac{1}{\Lambda^{2}}$ out front in equation (3.40) apparently suppresses $\delta S_{E E}^{(2)}$ in the limit $\Lambda \rightarrow \infty$. However, we must be careful in taking this limit because there is a possible enhancement from the $s$ integration inside $\mathcal{M}_{\overline{i j}}$. Indeed, naively sending $\Lambda \rightarrow \infty$ inside the integral in equation (3.42), we see that the $s$ integral diverges as $\int d s e^{s}$ in the limits $s \rightarrow \pm \infty$. We can extract these divergences by zooming in on the integral in these limits; this gives two contributions

$$
\mathcal{M}_{\overline{i j}}=\mathcal{M}_{\overline{i j}}^{\infty}+\mathcal{M}_{\overline{i j}}^{-\infty} .
$$

The contribution from $s \rightarrow \pm \infty$ can be extracted by changing variables to $\beta=\Lambda^{-2} e^{ \pm s}$

$$
\begin{aligned}
\mathcal{M}_{i j}^{ \pm \infty} & \simeq 2 \Lambda^{2} \int_{0}^{\infty} d \beta \frac{e^{ \pm 2 i\left(\tau_{a}-\tau_{b}\right)}}{\left(\left|x_{a}-x_{b}\right|^{2}-\beta e^{ \pm i\left(\tau_{a}-\tau_{b}\right)}\right)^{d+2}}\left(\begin{array}{cc}
1 & \pm i \\
\mp i & 1
\end{array}\right) \\
& =-\frac{2 \Lambda^{2}}{(d+1)} \frac{e^{ \pm i\left(\tau_{a}-\tau_{b}\right)}}{\left|x_{a}-x_{b}\right|^{2(d+1)}}\left(\begin{array}{cc}
1 & \pm i \\
\mp i & 1
\end{array}\right)
\end{aligned}
$$

Finally substituting the above into equation (3.40) and integrating over $\tau_{a}, \tau_{b}$, we get

$$
\delta S_{E E}^{(2)}=-\frac{2 \pi^{2} C_{T}}{(d+1)} \int d^{d-2} x_{a} d^{d-2} x_{b} \frac{1}{2}\left(\zeta^{1}\left(x_{a}\right) \zeta^{1}\left(x_{b}\right)+\zeta^{0}\left(x_{a}\right) \zeta^{0}\left(x_{b}\right)\right) \frac{1}{\left|x_{a}-x_{b}\right|^{2(d-1)}} .
$$

Reverting back to Lorentzian signature, we obtain

$$
\delta S_{E E}^{(2)}=-\frac{2 \pi^{2} C_{T}}{(d+1)} \int d^{d-2} x_{a} d^{d-2} x_{b} \frac{1}{2} \zeta^{\bar{i}}\left(x_{a}\right) \frac{\eta_{\overline{i j}}}{\left|x_{a}-x_{b}\right|^{2(d-1)}} \zeta^{\bar{j}}\left(x_{b}\right)
$$

which is our primary result. It still remains to be shown however, that the modular Hamiltonian term $\delta S_{E E}^{(1)}$ does not give additional contributions - we show this in the next section.

To end this section we would like to give some insight into the above calculation and in particular where the main contribution to the non-local part of the entanglement density is coming from. In words, the two stress tensor insertions start their lives close to the boundary of the entangling region (a distance $1 / \Lambda$ from $\partial A$ in the flat space metric.) 
However when we boost one of these operators by a rapidity of order $2 \ln \Lambda$, then the stress tensor gets liberated from $\partial A$ and moves into one of the null generators of $\partial \mathcal{D}(A)$, the boundary of the domain of dependence of $A$ - otherwise known as the Rindler horizon. Here the relevant integrated correlation function receives an enhancement of order $\Lambda^{2}$ in such a way that $\Lambda$ drops out of the final expression. Thus the main contribution to the entanglement density is coming from the correlation function of stress tensors inserted along null generators of $\partial \mathcal{D}(A)$. We find this result intriguing and intend to study this further in future works.

\subsection{The modular Hamiltonian term}

Now we return to the modular Hamiltonian term - in particular, the second term in equation (3.12) (the first term in (3.12) was studied in ref. [23] where it was shown to have no universal contributions). An alternative, less constructive, proof that this term vanishes is given in appendix E. Using the map $\varphi: \mathcal{H} \rightarrow \mathbb{R}^{d}$, this term pulls back to

$$
\delta S_{E E}^{(1)}=\frac{1}{8} \int_{\mathcal{H}_{\Lambda}} d \mu_{a} \int_{\mathcal{H}_{\Lambda}} d \mu_{b} \Omega^{-2}\left(y_{a}\right) h_{\alpha \beta}\left(y_{a}\right) \Omega^{-2}\left(y_{b}\right) h_{\gamma \delta}\left(y_{b}\right)\left\langle\widehat{T}^{\alpha \beta}\left(y_{a}\right) \widehat{T}^{\gamma \delta}\left(y_{b}\right) \widehat{H}_{E}\right\rangle_{\mathcal{H}_{\Lambda}}
$$

where recall that

$$
\left.\Omega^{-2} h_{\alpha \beta}=2 \nabla_{(\alpha} \xi_{\beta}\right)+\xi \cdot \partial \ln \left(\Omega^{2}\right) g_{\alpha \beta}^{\mathcal{H}} .
$$

Further, integrating by parts and using the diffeomorphism and trace Ward identities allows us to rewrite this as

$$
\begin{aligned}
\delta S_{E E}^{(1)}= & \frac{1}{8} \int_{\partial \mathcal{H}_{\Lambda}} d \bar{\mu}_{a} n^{\alpha}\left(y_{a}\right) \xi^{\beta}\left(y_{a}\right) \int_{\partial \mathcal{H}_{\Lambda}} d \bar{\mu}_{b} n^{\gamma}\left(y_{b}\right) \xi^{\delta}\left(y_{b}\right)\left\langle\widehat{T}_{\alpha \beta}\left(y_{a}\right) \widehat{T}_{\gamma \delta}\left(y_{b}\right) \widehat{H}_{E}\right\rangle_{\mathcal{H}_{\Lambda}} \\
& + \text { contact terms. }
\end{aligned}
$$

In appendix D, we will argue that the contact terms vanish in the limit $\Lambda \rightarrow \infty$, and so we focus here on the three-point function term. The modular Hamiltonian $\widehat{H}_{E}$ written on $S^{1} \times \mathbb{H}^{d-1}$ is simply the generator of $\tau$-translations

$$
\widehat{H}_{E}=2 \pi \int_{\mathbb{H}^{d-1}} \frac{d^{d-2} x_{c}^{i} d z_{c}}{z_{c}^{d-1}} \widehat{T}_{\tau \tau}\left(\tau_{c}, z_{c}, x_{c}^{i}\right)+\text { constant }
$$

where the integral above is on the constant $\tau=\tau_{c}$ slice. The constant term above drops out of all connected correlators. So the relevant correlation function in the present calculation is the three-point function of stress tensors on $\mathcal{H}$

$$
\begin{aligned}
\delta S_{E E}^{(1)}= & \frac{\pi}{4} \int_{\partial \mathcal{H}_{\Lambda}} d \bar{\mu}_{a} n^{\alpha}\left(y_{a}\right) \xi^{\beta}\left(y_{a}\right) \int_{\partial \mathcal{H}_{\Lambda}} d \bar{\mu}_{b} n^{\gamma}\left(y_{b}\right) \xi^{\delta}\left(y_{b}\right) \\
& \times \int_{\mathbb{H}^{d-1}} \frac{d^{d-2} x_{c}^{i} d z_{c}}{z_{c}^{d-1}}\left\langle\widehat{T}_{\alpha \beta}\left(y_{a}\right) \widehat{T}_{\gamma \delta}\left(y_{b}\right) \widehat{T}_{\tau \tau}\left(y_{c}\right)\right\rangle_{\mathcal{H}_{\Lambda}} .
\end{aligned}
$$

Once again, it is efficient to use the embedding space formalism to obtain this correlation function

$$
\left\langle\widehat{T}_{\alpha \beta}\left(y_{a}\right) \widehat{T}_{\gamma \delta}\left(y_{b}\right) \widehat{T}_{\tau \tau}\left(y_{c}\right)\right\rangle_{\mathcal{H}_{\Lambda}}=\frac{1}{8} \mathbb{P}_{\alpha \beta}^{A B}\left(P_{a}\right) \mathbb{P}_{\gamma \delta}^{C D}\left(P_{b}\right) \mathbb{P}_{\tau \tau}^{E F}\left(P_{c}\right) \frac{\partial^{2}}{\partial Z_{a}^{A} \partial Z_{a}^{B}} \frac{\partial^{2}}{\partial Z_{b}^{C} \partial Z_{b}^{D}} \frac{\partial^{2}}{\partial Z_{c}^{E} \partial Z_{c}^{F}} G^{(3)}
$$


where $^{8}$

$$
\begin{aligned}
& G^{(3)}\left(P_{a}, P_{b}, P_{c} ; Z_{a}, Z_{b}, Z_{c}\right) \\
& \quad=\left(-2 P_{a} \cdot P_{b}\right)^{-\frac{d+2}{2}}\left(-2 P_{b} \cdot P_{c}\right)^{-\frac{d+2}{2}}\left(-2 P_{c} \cdot P_{a}\right)^{-\frac{d+2}{2}} \sum_{m=1}^{5} \alpha_{m} A_{m}\left(V_{I}, H_{J K}\right) .
\end{aligned}
$$

The $\left\{A_{m}\right\}$ 's are conformally invariant structures — polynomials made from six basic building blocks [42]

$$
\begin{aligned}
H_{I J} & =-2\left(\left(Z_{I} \cdot Z_{J}\right)\left(P_{I} \cdot P_{J}\right)-\left(Z_{I} \cdot P_{J}\right)\left(Z_{J} \cdot P_{I}\right)\right) \\
V_{I} & =\frac{\left(Z_{I} \cdot P_{J}\right)\left(P_{I} \cdot P_{K}\right)-\left(Z_{I} \cdot P_{K}\right)\left(P_{I} \cdot P_{J}\right)}{P_{J} \cdot P_{K}}
\end{aligned}
$$

where the triplet $(I, J, K)$ is a cyclic permutation of $(a, b, c)$. The allowed structures are ${ }^{9}$

$$
\begin{aligned}
& A_{1}=V_{a}^{2} V_{b}^{2} V_{c}^{2} \\
& A_{2}=H_{a b} V_{a} V_{b} V_{c}^{2}+H_{b c} V_{b} V_{c} V_{a}^{2}+H_{c a} V_{c} V_{a} V_{b}^{2} \\
& A_{3}=V_{a} H_{a b} H_{b c} V_{c}+V_{b} H_{b c} H_{c a} V_{a}+V_{c} H_{c a} H_{a b} V_{b} \\
& A_{4}=H_{a b}^{2} V_{c}^{2}+H_{b c}^{2} V_{a}^{2}+H_{c a}^{2} V_{b}^{2} \\
& A_{5}=H_{a b} H_{b c} H_{c a} .
\end{aligned}
$$

The coefficients $\alpha_{m}$ in (3.54) are not all independent - imposing the conservation condition on the stress tensors for non-coincident points gives the constraints

$$
\begin{aligned}
& C_{1}\left(\alpha_{m}\right) \equiv-2 \alpha_{1}+4 \alpha_{2}+\left(\frac{d^{2}}{2}+d-4\right) \alpha_{3}-d(d+2) \alpha_{4}=0 \\
& C_{2}\left(\alpha_{m}\right) \equiv \alpha_{2}-\frac{d+2}{2} \alpha_{3}+2 d \alpha_{4}+\frac{1}{2}\left(4-d^{2}\right) \alpha_{5}=0 .
\end{aligned}
$$

This fixes two of the coefficients in terms of the rest, leaving three independent coefficients. ${ }^{10}$

In general, computing the integrals in (3.52) over the hyperbolic slice at $\tau=\tau_{c}$ is a difficult task. However, the following observation makes this computation tractable the modular Hamiltonian is a conserved charge and so we are free to move it in $\tau$. One therefore expects $\delta S_{E E}^{(1)}$ to be independent of $\tau_{c}$. One might worry about potential crossing contributions to $\delta S_{E E}^{(1)}$ when we move the modular Hamiltonian across one of the other stress-tensor insertions, but it can be checked explicitly that these vanish in the limit

\footnotetext{
${ }^{8}$ Additionally, there are further contact terms in the three-point function which are required by the trace Ward identity. However, these contact terms do not contribute in the limit $\Lambda \rightarrow \infty$, and so we do not show them here explicitly.

${ }^{9}$ In three dimensions there is also potentially a parity odd structure $[43,44]$ that we did not write down. However it is easy to argue that such a term cannot contribute to the non-local part of the entanglement density based on symmetries and unitarity — there is no parity odd term that we could add to (1.6) or (1.7) which preserves the appropriate conformal symmetries and the strong subaddativity constraint.

${ }^{10}$ Imposing the conservation equation in the coincident limit fixes a linear combination of these three coefficients in terms of $C_{T}$ [41].
} 


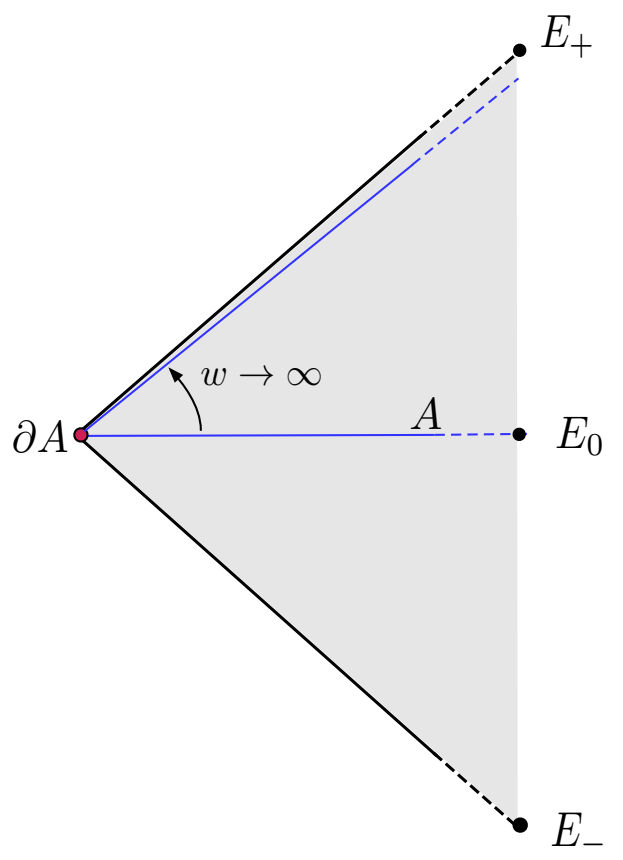

Figure 3. The shaded region is the domain of dependence $\mathcal{D}(A)$. Analytically continuing in $w=e^{i \tau_{c}}$ and sending $w \rightarrow \infty$ sends the modular Hamiltonian (integrated over the blue line) to the future null boundary of $\mathcal{D}(A)$. Also shown are the future and past tips $E_{ \pm}$of $\mathcal{D}(A)$ and the point at spacelike infinity $E_{0}$.

$\Lambda \rightarrow \infty$ (see appendix D). Therefore, in the complex $w=e^{i \tau_{c}}$ plane, $\delta S_{E E}^{(1)}$ can be extended to an analytic function which is constant along the unit circle $|w|=1$, and hence a constant on the entire $w$ plane. We can therefore use this to our advantage by computing $\delta S_{E E}^{(1)}$ at a special point such as $w=0$ or $w=\infty$. Physically, these two-points correspond to light-cone limits: $w \rightarrow 0$ corresponds to writing the modular Hamiltonian as an integral over the past null boundary of the domain of dependence $\mathcal{D}(A)$ (or the past Rindler horizon for brevity), while $w \rightarrow \infty$ corresponds to writing it as an integral over the future Rindler horizon (see figure 3 ). We take $w \rightarrow \infty$ in what follows.

The computation simplifies dramatically in this limit. To see this is more detail, define the points

$$
E_{ \pm}=(0,1, \pm i, 0, \cdots, 0), \quad E_{0}=(1,0,0,-1,0 \cdots, 0)
$$

in the embedding space. These points have an interesting physical interpretation - (the rays corresponding to) $E_{ \pm}$form the future and past tips of the light cone comprising the boundary of the domain of dependence of $A$, while (the ray corresponding to) $E_{0}$ constitutes the point at spacelike infinity, or equivalently the point at infinity in the Poincaré coordinates of hyperbolic space $\mathbb{H}^{d-1}$. We note the relations

$$
E_{+} \cdot E_{+}=E_{-} \cdot E_{-}=0, \quad E_{+} \cdot E_{-}=2, \quad E_{0}^{2}=0, \quad E_{0} \cdot E_{ \pm}=0 .
$$


The relevant projectors in (3.53) (with $z_{a, b}=\frac{1}{\Lambda}$ ) can be written in terms of these points as ${ }^{11}$

$$
\begin{aligned}
\mathbb{P}_{\tau z}^{A B}\left(P_{a, b}\right)= & \frac{i \Lambda}{4}\left(\left(P_{a, b}^{+}\right)^{2} E_{-}^{A} E_{-}^{B}-\left(P_{a, b}^{-}\right)^{2} E_{+}^{A} E_{+}^{B}\right)+\frac{1}{2 i}\left(P_{a, b}^{-} E_{+}^{(A}-P_{a, b}^{+} E_{-}^{(A}\right) E_{0}^{B)} \\
\mathbb{P}_{z z}^{A B}\left(P_{a, b}\right)= & \frac{\Lambda^{2}}{4}\left(\left(P_{a, b}^{+}\right)^{2} E_{-}^{A} E_{-}^{B}+\left(P_{a, b}^{-}\right)^{2} E_{+}^{A} E_{+}^{B}+2 E_{+}^{(A} E_{-}^{B)}-\frac{4}{d} \eta^{A B}\right) \\
& +\Lambda\left(P_{a, b}^{-} E_{+}^{(A}+P_{a, b}^{+} E_{-}^{(A}\right) E_{0}^{B)}+E_{0}^{A} E_{0}^{B} \\
\lim _{w \rightarrow \infty} \mathbb{P}_{\tau \tau}^{A B}\left(P_{c}\right)= & -\frac{w^{2}}{4}\left(E_{\mp}^{A} E_{\mp}^{B}+O\left(w^{-1}\right)\right)
\end{aligned}
$$

where $P_{a, b}^{ \pm}=P_{a, b} \cdot E^{ \pm}$. The only other ingredient required to compute $\delta S_{E E}^{(1)}$ is the following generic integral

$I\left(n_{+}, n_{-}, n_{0} \mid m_{a}, m_{b}\right)=\int_{\mathbb{H}^{d-1}} d Y_{c}\left(-2 P_{a} \cdot P_{c}\right)^{-m_{a}}\left(-2 P_{b} \cdot P_{c}\right)^{-m_{b}}\left(E_{+} \cdot P_{c}\right)^{n_{+}}\left(E_{-} \cdot P_{c}\right)^{n_{-}}\left(E_{0} \cdot P_{c}\right)^{n_{0}}$

where $d Y_{c}$ is the appropriate integration measure over $\mathbb{H}^{d-1}$. Precisely in the limit $w \rightarrow$ $\infty$, this integral simplifies greatly and can be written in terms of a single integral (see appendix E)

$$
\begin{aligned}
I\left(n_{+}, n_{-}, n_{0} \mid m_{a}, m_{b}\right)= & -\frac{k}{w^{2}} \delta_{n_{-}, 0} \int_{0}^{\infty} d \lambda \frac{\lambda^{m_{a}-m_{b}-1}}{\left(\lambda P_{a}^{\mp}+\lambda^{-1} P_{b}^{\mp}\right)^{n_{+}+2}} \frac{\left(-\lambda\left(E_{0} \cdot Y_{a}\right)-\lambda^{-1}\left(E_{0} \cdot Y_{b}\right)\right)^{n_{0}}}{\left(\lambda^{2}+\frac{1}{\lambda^{2}}-2 Y_{a} \cdot Y_{b}\right)^{\frac{d}{2}-1+n_{0}}} \\
& +O\left(w^{-3}\right)
\end{aligned}
$$

where $Y_{a, b}$ are the embedding space coordinates for $\mathbb{H}^{d-1}$, and the constant $k$ is given by

$$
k=2 \pi^{\frac{d-2}{2}}(-1)^{n_{0}+n_{+}} \frac{\Gamma\left(\frac{d}{2}-1+n_{0}\right) \Gamma\left(n_{+}+2\right)}{\Gamma\left(m_{a}\right) \Gamma\left(m_{b}\right)} .
$$

Putting everything together, one finds

$$
\delta S_{E E}^{(1)}=\frac{\pi}{4} \int_{\partial \mathcal{H}_{\Lambda}} d \bar{\mu}_{a} \int_{\partial \mathcal{H}_{\Lambda}} d \bar{\mu}_{b} \hat{\xi}^{\bar{i}}\left(y_{a}\right) \mathcal{N}_{\overline{i j}} \hat{\xi}^{\bar{j}}\left(y_{b}\right)
$$

where the matrix $\mathcal{N}_{\overline{i j}}$ can be explicitly computed as a series expansion in $\Lambda^{2}$. For instance,

$$
\mathcal{N}_{\tau \tau}=\frac{c}{\left|x_{a}-x_{b}\right|^{2(d-1)}}\left(\Lambda^{2}\left|x_{a}-x_{b}\right|^{2} C_{1}\left(\alpha_{m}\right)-d\left[2-\cos \left(\tau_{a}-\tau_{b}\right)\right] C_{1}\left(\alpha_{m}\right)+O\left(\Lambda^{-2}\right)\right)
$$

where

$$
c=\frac{(-1)^{d} \pi^{d / 2-1}}{d^{2}(d+2)^{2}(d+4) \Gamma\left(\frac{d}{2}\right)} .
$$

Now comes the surprising part: the terms which could potentially survive in the $\Lambda \rightarrow \infty$ limit come multiplied by the function $C_{1}\left(\alpha_{m}\right)$ defined in (3.62), which vanishes by the conservation constraints. The same is true for all the components of the matrix $\mathcal{N}-$ the

\footnotetext{
${ }^{11}$ The function $G^{(3)}$ in $(3.54)$, by construction, satisfies the transversality conditions $P_{a, b, c} \cdot \frac{\partial}{\partial Z_{a, b, c}} G^{(3)}=0$, and we have used these to simplify the projectors.
} 
potentially non-trivial terms in the $\Lambda \rightarrow \infty$ limit are all proportional to linear combinations of $C_{1}\left(\alpha_{m}\right)$ and $C_{2}\left(\alpha_{m}\right)$. Therefore,

$$
\lim _{\Lambda \rightarrow \infty} \mathcal{N}_{\overline{i j}}=0 .
$$

This completes our proof that the modular Hamiltonian term does not give additional contributions to the non-local part of the entanglement density.

\subsection{Spherical case}

So far, we have presented the calculation for the non-local part of the entanglement density in the case of planar entangling surfaces. It is possible to repeat the above calculation for spherical entangling surfaces, but here we will obtain the corresponding result more directly by making use of the conformal transformation $\psi: \mathbb{R}^{1, d-1} \rightarrow \mathbb{R}^{1, d-1}$ given by

$$
\psi^{\mu}(\boldsymbol{X})=\frac{\boldsymbol{X}^{\mu}-(\boldsymbol{X} \cdot \boldsymbol{X}) C^{\mu}}{1-2(C \cdot \boldsymbol{X})+(\boldsymbol{X} \cdot \boldsymbol{X})(C \cdot C)}+2 R^{2} C^{\mu}
$$

where we have used $\boldsymbol{X}^{\mu}=\left(X^{0}, X^{1}, X^{i}\right)$ as coordinates on the domain of $\psi$, and $C=\left(0, \frac{1}{2 R}, 0, \cdots, 0\right)$. This is a conformal transformation because $\psi_{*} \eta=\omega^{2} \eta$, with the conformal factor $\omega$ given by

$$
\omega(\boldsymbol{X})=\frac{1}{1-2(C \cdot \boldsymbol{X})+(\boldsymbol{X} \cdot \boldsymbol{X})(C \cdot C)} .
$$

If we use global coordinates $\boldsymbol{x}^{\mu}=\left(x^{0}, \mathbf{x}\right)$ to cover the image of this map, then it is a simple matter to check the following statements: (i) $\psi$ maps the Cauchy surface $X^{0}=0$ to the Cauchy surface $x^{0}=0$, (ii) if $A$ is the half-space $X^{1} \geq 0$ on the Cauchy surface $X^{0}=0$, then $B=\psi(A)$ is the ball-shaped region $\mathrm{x}^{2} \leq R^{2}$ on the Cauchy surface $x^{0}=0$, (iii) $\psi$ maps the domain of dependence of $A$ to the domain of dependence of $B$. Consequently, we can compute the entanglement density for the ball-shaped region $B$ by pushing forward the deformation vector field $\zeta_{\partial B}\left(\Omega^{i}\right)$ (where $\Omega^{i}$ are coordinates on the sphere $\partial B$ ) by $\psi^{-1}$

$$
\zeta_{\partial A}^{\mu}\left(X^{i}\right)=\frac{\partial \boldsymbol{X}^{\mu}}{\partial \boldsymbol{x}^{\nu}} \zeta_{\partial B}^{\nu}\left(\Omega^{i}\right)
$$

and then computing the corresponding entanglement density for the half-space $A$

$$
\delta^{(2)} S_{E E}(B)=\delta^{(2)} S_{E E}(A)=-\frac{2 \pi^{2} C_{T}}{d+1} \int d^{d-2} X_{a} d^{d-2} X_{b} \frac{1}{2} \zeta_{\partial A}^{\bar{i}}\left(X_{a}\right) \zeta_{\partial A}^{\bar{j}}\left(X_{b}\right) \frac{\eta_{\overline{i j}}}{\left|X_{a}-X_{b}\right|^{2(d-1)}}
$$

Since the map $\psi$ is a conformal transformation, the Jacobian factor in (3.77) can be written as

$$
\frac{\partial \boldsymbol{X}^{\mu}}{\partial \boldsymbol{x}^{\nu}}=\omega^{-1}(\boldsymbol{X}) \mathcal{R}^{\mu}{ }_{\nu}(\psi)
$$

where $\mathcal{R}$ is a rotation. Since $\zeta_{\partial B}$ lies in the plane perpendicular to the entangling surface $\mathrm{x}^{2}=R^{2}$, it follows that $\zeta_{\partial A}$ also lies in the plane perpendicular to the surface $X^{1}=0$. 
Further, by rotation symmetry along $\partial B$ (or equivalently, translation symmetry along its inverse image $\partial A$ ), we deduce

$$
\eta_{\overline{i j}} \zeta_{\partial A}^{\bar{i}}\left(X_{a}\right) \zeta_{\partial A}^{\bar{j}}\left(X_{b}\right)=\omega^{-1}\left(0,0, X_{a}\right) \omega^{-1}\left(0,0, X_{b}\right) \eta_{\overline{i j}} \zeta_{\partial B}^{\bar{i}}\left(\Omega_{a}\right) \zeta_{\partial B}^{\bar{j}}\left(\Omega_{b}\right)
$$

Finally, using the relations

$$
\begin{aligned}
R^{d-2} d^{d-2} \Omega_{a} & =\omega^{d-2}\left(0,0, X_{a}\right) d^{d-2} X_{a} \\
\left(\boldsymbol{x}_{a}-\boldsymbol{x}_{b}\right)^{2} & =\omega\left(\boldsymbol{X}_{a}\right) \omega\left(\boldsymbol{X}_{b}\right)\left(\boldsymbol{X}_{a}-\boldsymbol{X}_{b}\right)^{2}
\end{aligned}
$$

we obtain

$$
\delta^{(2)} S_{E E}(B)=-\frac{2 \pi^{2} C_{T}}{R^{2}(d+1)} \int d^{d-2} \Omega_{a} d^{d-2} \Omega_{b} \frac{1}{2} \zeta_{\partial B}^{\bar{i}}\left(\Omega_{a}\right) \zeta_{\partial B}^{\bar{j}}\left(\Omega_{b}\right) \frac{\eta_{\overline{i j}}}{\left|\Omega_{a}-\Omega_{b}\right|^{2(d-1)}}
$$

which is the result for the entanglement density for spheres.

\section{Applications}

In this section we will present some applications of our formula for the entanglement density. In section 4.1, we will prove the conjectured universality of the corner term contribution to entanglement entropy in $d=3[24,25]$. In section 4.2 , we will prove the Mezei formula for the shape dependence of entanglement entropy across deformed spheres, which was conjectured based on holographic calculations in [26]. In [27], the Mezei formula was used to deduce further universality results for corner terms in higher dimensions. Our proof of the Mezei formula thus also establishes the universality of corner terms in higher dimensions.

\subsection{Corner terms in $d=3$}

The entanglement entropy of a general subregion in the vacuum state of a $d=3$ CFT takes the general form

$$
S_{E E}=a_{1} \frac{\ell}{\delta}-a(\theta) \ln \frac{\ell}{\delta}+O(1)
$$

where $\delta$ is a short-distance cutoff and $\ell$ is a length scale associated with the size of the subregion. The first term above is the area-law term, while the second term, which is universal, only appears in cases when the subregion has a sharp corner with opening angle $\theta$, henceforth referred to as the corner term. It has been conjectured based on holographic, free-field and numerical calculations, that in the smooth limit $\theta \rightarrow \pi$, the corner term in any $d=3$ CFT behaves as

$$
a(\theta)=\frac{\pi^{2} C_{T}}{24}(\theta-\pi)^{2}+\cdots
$$

where $C_{T}$, once again, is the coefficient appearing in the two-point function of stress tensors in that CFT. Here, we will show that our formula for the entanglement density directly reproduces equation (4.2). 
We start with our formula for the planar case in $d=3$ :

$$
\delta^{(2)} S_{E E}=-\frac{\pi^{2} C_{T}}{4} \int_{-\infty}^{\infty} d x_{a} \int_{-\infty}^{\infty} d x_{b} \frac{\chi\left(x_{a}\right) \chi\left(x_{b}\right)}{\left(x_{a}-x_{b}\right)^{4}}
$$

and consider the special shape deformation:

$$
\chi(x)= \begin{cases}0 & |x|>L \\ \frac{\alpha}{2 L}\left(L^{2}-x^{2}\right) & |x|<L\end{cases}
$$

which has two sharp corners at $x= \pm L$ with opening angle $\theta=(\pi-\alpha)$. The two corners will both lead to independent logarithmic divergences which we can then isolate. We choose this form because it does not have any IR issues and because it is easy to work with analytically. ${ }^{12}$

Note that in order to do the integrals in (4.3) we are forced to confront UV divergences when the two-points $x_{a}$ and $x_{b}$ come together. This is then related to local contact terms in the entanglement density that we have so far avoided discussing. These terms are also related to the usual UV divergence of EE (that is the area law piece for $d=3$ shown in (4.1).) An efficient way to deal with these contact terms is to use dimensional regularization where the absence of a scale in the regulator means that we will only ever see logarithmic divergences (which would then show up as $1 /(d-3)$ poles.) Since we do not expect logarithmic divergences in $d=3$ in the absence of sharp corners, this is then a good way of isolating the term of interest. To this end, we consider an entangling surface in a $d$-dimensional CFT with the shape determined by (4.4) independent of the other $d-3$ transverse directions. At second order the change in entanglement entropy is then:

$$
\delta^{(2)} S_{E E}=-\frac{\pi^{2} \alpha^{2} C_{T}}{4(d+1) L^{2}} V_{d-3} \frac{\pi^{(d-3) / 2} \Gamma\left(\frac{d+1}{2}\right)}{\Gamma(d-1)} \int_{-L}^{L} d x_{a} \int_{-L}^{L} d x_{b} \frac{\left(L^{2}-x_{a}^{2}\right)\left(L^{2}-x_{b}^{2}\right)}{\left|x_{a}-x_{b}\right|^{d+1}}
$$

where $V_{d-3}=\mu^{d-3}$ is the volume of the transverse space. This last integral can easily be done (and converges for $d<0$ ) giving:

$$
\delta^{(2)} S_{E E}=-\frac{\pi^{2} \alpha^{2} C_{T}(d-1) \Gamma\left(\frac{d-5}{2}\right)}{2 d(d+1)(d-2) \Gamma(d-1)}\left(\frac{\sqrt{\pi} \mu}{2 L}\right)^{d-3} .
$$

Taking the limit $d \rightarrow 3$ we find the desired pole and logarithmic behavior:

$$
\delta^{(2)} S_{E E}=\frac{C_{T} \pi^{2} \alpha^{2}}{12}\left(\frac{1}{d-3}-\log (2 L /(\sqrt{\pi} \mu))+\frac{\gamma}{2}-\frac{19}{12}+\mathcal{O}(d-3)\right) .
$$

Since we had two corners with equal opening angles we can infer that:

$$
a(\theta)=\frac{\pi^{2} C_{T}}{24}(\pi-\theta)^{2}
$$

which proves the conjecture of $[24,25]$. We have thus shown that in $d=3$ the logarithmic corner term in the entanglement entropy is entirely captured by the non-local part of the entanglement density. Presumably similar remarks/proofs hold for higher dimensional cones as conjectured recently in [27] using the Mezei formula.

\footnotetext{
${ }^{12}$ A somewhat similar form was used in [27] to show that the Mezei formula reproduces the corner term.
} 


\subsection{Mezei formula}

Next we turn to proving the Mezei formula [26] for the entanglement entropy across deformed spheres at second order in the shape deformation. Once again in this section, we will only be interested in spatial deformations. Let us expand the shape deformation $\chi(\Omega)$ in terms of real hyperspherical harmonics on the entangling surface $S^{d-2}$

$$
\chi(\Omega)=\sum_{\ell, m_{1}, \cdots, m_{d-3}} a_{\ell, m_{1}, \cdots, m_{d-3}} Y_{\ell, m_{1}, \cdots, m_{d-3}}(\Omega) .
$$

Based on holographic calculations in a large class of models, it was conjectured in [26] that the universal contribution to the entanglement entropy at second order in the deformation is given by

$$
S_{E E}^{(2)}=C_{T} \frac{\pi^{\frac{d+2}{2}}(d-1)}{2^{d-2} \Gamma(d+2) \Gamma(d / 2)} \sum_{\ell, m_{1}, \cdots, m_{d-3}} a_{\ell, m_{1}, \cdots, m_{d-3}}^{2} \prod_{k=1}^{d}(\ell+k-2) \times\left\{\begin{array}{ll}
(-1)^{\frac{d-1}{2}} \frac{\pi}{2} & d \text { odd } \\
(-1)^{\frac{d-2}{2}} \ln \frac{R}{\delta} & d \text { even }
\end{array} .\right.
$$

The positivity of the overall coefficient implies that the sphere is a local minimum for (the universal part of) entanglement entropy across all shapes with the same topology. Having derived the entanglement density for spheres from purely CFT considerations, we are now in a position to prove this conjecture. We start with our expression for the sphere entanglement density (setting $R=1$ for convenience)

$$
S_{E E}^{(2)}=-\frac{\pi^{2} C_{T}}{(d+1) 2^{d-1}} \int d^{d-2} \Omega_{a} d^{d-2} \Omega_{b} \chi\left(\Omega_{a}\right) \chi\left(\Omega_{b}\right) \frac{1}{\left(1-\Omega_{a} \cdot \Omega_{b}\right)^{d-1}} .
$$

Substituting equation (4.9), we obtain

$$
\begin{aligned}
S_{E E}^{(2)}= & -\frac{\pi^{2} C_{T}}{(d+1) 2^{d-1}} \sum_{\ell^{a}, m_{1}^{a}, \cdots, \ell^{b}, m_{1}^{b}, \cdots} a_{\ell^{a}, m_{1}^{a}, \cdots} a_{\ell^{b}, m_{1}^{b}, \cdots} \\
& \times \int d^{d-2} \Omega_{a} d^{d-2} \Omega_{b} \frac{Y_{\ell^{a}, m_{1}^{a}, \cdots, m_{d-3}^{a}}\left(\Omega_{a}\right) Y_{\ell^{b}, m_{1}^{b}, \cdots, m_{d-3}^{b}}\left(\Omega_{b}\right)}{\left(1-\Omega_{a} \cdot \Omega_{b}\right)^{d-1}} .
\end{aligned}
$$

From rotation invariance, it is evident that the integral above takes the form

$$
\int d^{d-2} \Omega_{a} d^{d-2} \Omega_{b} \frac{Y_{\ell^{a}, m_{1}^{a}, \cdots, m_{d-3}^{a}}\left(\Omega_{a}\right) Y_{\ell^{b}, m_{1}^{b}, \cdots, m_{d-3}^{b}}\left(\Omega_{b}\right)}{\left(1-\Omega_{a} \cdot \Omega_{b}\right)^{d-1}}=c\left(\ell^{a}\right) \delta_{\ell^{a} \ell^{b}} \delta_{m_{1}^{a}, m_{1}^{b}} \cdots \delta_{m_{d-3}^{a}, m_{d-3}^{b}} .
$$

The constant on the right hand side can in turn be written as

$$
c(\ell)=\frac{1}{\operatorname{dim}\left(H_{\ell}\right)} \int d^{d-2} \Omega_{a} d^{d-2} \Omega_{b} \sum_{m_{1}, \cdots, m_{d-3}} \frac{Y_{\ell, m_{1}, \cdots, m_{d-3}}\left(\Omega_{a}\right) Y_{\ell, m_{1}, \cdots, m_{d-3}}\left(\Omega_{b}\right)}{\left(1-\Omega_{a} \cdot \Omega_{b}\right)^{d-1}}
$$

where $H_{\ell}$ is the space of all harmonics of order $\ell$. In order to explicitly compute $c(\ell)$, we can use the higher-dimensional analog of the addition theorem for spherical harmonics ${ }^{13}$

$$
\sum_{m_{1}, \cdots, m_{d-3}} Y_{\ell, m_{1}, \cdots, m_{d-3}}\left(\Omega_{a}\right) Y_{\ell, m_{1}, \cdots, m_{d-3}}\left(\Omega_{b}\right)=\frac{\operatorname{dim}\left(H_{\ell}\right)}{\operatorname{vol}\left(S^{d-2}\right)} \frac{C_{\ell}^{\left(\frac{d-3}{2}\right)}\left(\Omega_{a} \cdot \Omega_{b}\right)}{C_{\ell}^{\left(\frac{d-3}{2}\right)}(1)}
$$

\footnotetext{
${ }^{13}$ We have normalized the hyperspherical harmonics as $\int_{S^{d-2}} d^{d-2} \Omega\left(Y_{\ell, m_{1}, \cdots, m_{d-3}}(\Omega)\right)^{2}=1$.
} 
where $C_{\ell}^{(n)}(x)$ is the Gegenbauer polynomial. This allows us to perform all but one of the integrals in (4.14) to obtain

$$
c(\ell)=\lim _{z \rightarrow 1^{+}} \frac{\operatorname{vol}\left(S^{d-3}\right)}{C_{\ell}^{\left(\frac{d-3}{2}\right)}(1)} \int_{0}^{\pi} d \theta \sin ^{d-3} \theta \frac{C_{\ell}^{\left(\frac{d-3}{2}\right)}(\cos \theta)}{(z-\cos \theta)^{d-1}} .
$$

Note that we have also introduced the regulator $z$ above to control the divergences which arise in the $\theta \rightarrow 0$ (coincident) limit. Fortunately, there exists a closed form expression for the above $\theta$ integral $[45,46]$

$$
\int_{0}^{\pi} d \theta(\sin \theta)^{D-1} \frac{C_{\ell}^{\left(\frac{D-1}{2}\right)}(\cos \theta)}{(z-\cos \theta)^{D+1}}=\frac{e^{-i(D / 2+1) \pi} \sqrt{\pi} \Gamma(D+\ell-1)}{2^{D / 2-2} \Gamma(\ell+1) \Gamma\left(\frac{D-1}{2}\right) \Gamma(D+1)} \frac{1}{\left(z^{2}-1\right)^{\frac{D+2}{4}}} Q_{D / 2+\ell-1}^{D / 2+1}(z)
$$

where the associated Legendre function $Q_{D / 2+\ell-1}^{D / 2+1}(z)$ is defined in terms of the hypergeometric function as

$$
Q_{D / 2+\ell-1}^{D / 2+1}(z)=\frac{e^{i \pi(D / 2+1)} \sqrt{\pi} \Gamma(D+\ell+1)}{2^{D / 2+\ell} \Gamma\left(D / 2+\ell+\frac{1}{2}\right)} \frac{z^{1-\ell}}{\left(z^{2}-1\right)^{\frac{D+2}{4}}}{ }_{2} F_{1}\left(\frac{\ell-1}{2}, \frac{\ell}{2} ; \frac{D}{2}+\ell+\frac{1}{2} ; \frac{1}{z^{2}}\right) .
$$

Using these expressions, we obtain ${ }^{14}$

$$
\begin{aligned}
c(\ell)= & \lim _{z \rightarrow 1^{+}} \frac{2 \pi^{\frac{d}{2}}}{\Gamma\left(\frac{d-2}{2}\right)} \frac{\Gamma(d+\ell-1) \Gamma(d-3)}{2^{d+\ell-4} \Gamma\left(\frac{d-3}{2}\right) \Gamma(d-1) \Gamma\left(d / 2+\ell-\frac{1}{2}\right)} \frac{z^{1-\ell}}{\left(z^{2}-1\right)^{\frac{d}{2}}} \\
& \times{ }_{2} F_{1}\left(\frac{\ell-1}{2}, \frac{\ell}{2} ; \frac{d}{2}+\ell-\frac{1}{2} ; \frac{1}{z^{2}}\right) .
\end{aligned}
$$

All that remains to be done is to take the limit $z \rightarrow 1^{+}$. Let us first consider $d$ odd; in this case the hypergeometric function behaves as

$$
{ }_{2} F_{1}\left(\frac{\ell-1}{2}, \frac{\ell}{2} ; \frac{d}{2}+\ell-\frac{1}{2} ; 1-\epsilon\right)=\left(a_{0}+a_{1} \epsilon+a_{2} \epsilon^{2}+\cdots\right)+\epsilon^{d / 2}\left(b_{0}+b_{1} \epsilon+b_{2} \epsilon^{2}+\cdots\right)
$$

Going back to (4.19), we see that $c(\ell)$ is divergent in the limit $z \rightarrow 1^{+}$. However, these divergences, as before, are associated with the coincident limit $\Omega_{a} \rightarrow \Omega_{b}$. A proper treatment of these divergences would require knowledge of contact terms in the entanglement density, which we are not in control of. However, we can extract the universal (cutoff independent) term in (4.19), which comes from the $\epsilon^{d / 2}$ term in the expansion of the hypergeometric function close to $z=1$, where the corresponding coeffcient $b_{0}$ is given by

$$
b_{0}=(-1)^{\frac{d+1}{2}} \frac{\Gamma\left(\frac{d}{2}+\ell-\frac{1}{2}\right)}{\Gamma\left(\frac{\ell}{2}\right) \Gamma\left(\frac{\ell-1}{2}\right) \Gamma\left(\frac{d}{2}+1\right)} \pi .
$$

\footnotetext{
${ }^{14}$ The careful reader might observe that the functional form (in $z$ ) appearing above is very closely related to the (deformed) Ryu-Takayanagi surface, with $\sin \Theta=\frac{1}{z}$ playing the role of the bulk coordinate defined in [26]. This motivates the identification $\epsilon=\frac{z^{2}-1}{z^{2}} \sim\left(\frac{\delta}{R}\right)^{2}$.
} 


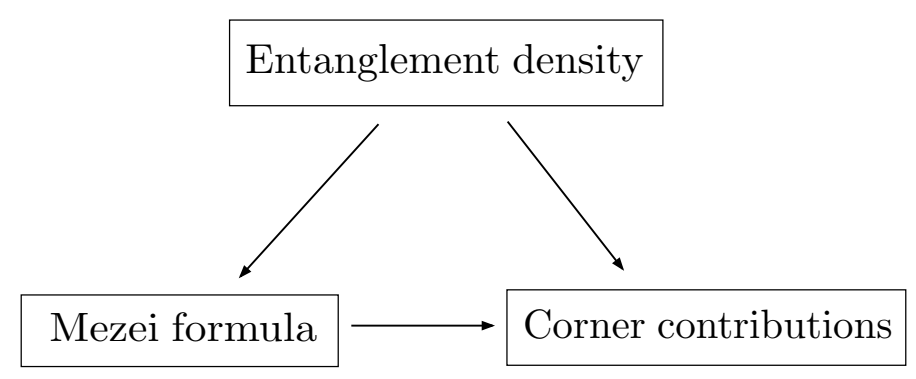

Figure 4. The triangle of recent studies on the shape dependence of entanglement entropy in CFTs. The arrows denote implications.

For $d$ even, the expansion of the hypergeometric function contains a logarithmic term $\epsilon^{d / 2} \ln \epsilon$ which then gives the universal contribution to the entanglement entropy, and whose coefficient is given by

$$
\tilde{b}_{0}=(-1)^{\frac{d-2}{2}} \frac{\Gamma\left(\frac{d}{2}+\ell-\frac{1}{2}\right)}{\Gamma\left(\frac{\ell}{2}\right) \Gamma\left(\frac{\ell-1}{2}\right) \Gamma\left(\frac{d}{2}+1\right)} .
$$

Finally, putting everything together and simplifying gives

$S_{E E}^{(2)}=C_{T} \frac{\pi^{\frac{d+2}{2}}(d-1)}{2^{d-2} \Gamma(d+2) \Gamma(d / 2)} \sum_{\ell, m_{1}, \cdots, m_{d-3}} a_{\ell, m_{1}, \cdots, m_{d-3}}^{2} \prod_{k=1}^{d}(\ell+k-2) \times \begin{cases}(-1)^{\frac{d-1}{2}} \frac{\pi}{2} & d \text { odd } \\ (-1)^{\frac{d-2}{2}} \ln \frac{R}{\delta} & d \text { even }\end{cases}$

which is precisely the formula conjectured in [26].

As mentioned previously, in [27] the Mezei conjecture was used to compute the universal corner term contributions to entanglement entropy in higher dimensions. Since we have now explicitly proved the Mezei conjecture, this also completes the derivation of the higher dimensional corner terms in [27].

\section{Discussion}

We have presented a proof of the universality of the non-local part of entanglement density in any CFT in any dimension. The form of the entanglement density is fixed by conformal invariance and the overall coefficient is determined by $C_{T}$. We have also shown that this universality fits into a triangle of results that have been the focus of recent studies/conjectures on the shape dependence of CFT entanglement entropy and that we summarize in figure 4.

We have a good understanding of the mechanism behind this universality. The calculation presented here and previous works $[6,21]$ studied entanglement essentially in conformal perturbation theory, writing the answer in terms of $n$ point correlation functions on flat space up to some order $n$. Conformal invariance fixes the low point correlation functions that go into the calculation. As we push these calculations to higher order in the expansion parameter, four-point functions and higher will appear and we expect universality to break down. At this point one might impose more restrictive conditions on the CFT that are expected of a theory with a gravitational dual - large- $N$ factorization and an appropriate sparseness condition on the low-lying spectrum of operator dimensions [47] - after which 
we would expect universality to re-emerge. Ryu-Takayangi taught us the surprising result that all CFTs with classical gravity duals have the same vacuum entanglement structure. We are far from being able to prove such a statement, ${ }^{15}$ but these calculations represent a first step.

It is interesting to note that even before we reach the stage where universality breaks down, entanglement in this perturbative framework already displays rich features that are expected of a CFT with a holographic dual [6]. The relative entropy contribution studied in section 3.1 arises from an integral over an operator located inside the domain of dependence of $A$, which reminds us of smearing functions that are used to construct local bulk fields [49]. It is this feature of the CFT calculation that we think is responsible for probing deep into the bulk of an emergent AdS dual to measure the change in area of the minimal surface.

We now discuss some possible generalizations that one may pursue. Firstly it would be of interest to study the Renyi generalization of entanglement density (see [50] for related discussion). For example using the mutual information definition of entanglement density (1.4), we can imagine simply generalizing this by taking $I \rightarrow I_{n}$ where $I_{n}$ is called the Renyi mutual information. This captures the non-local part of the Renyi entanglement density which is then a UV finite quantity. It is not hard to argue, based on conformal invariance alone, that Renyi entanglement density should take the same form as regular entanglement density:

$$
S_{\overline{i j}, \text { non-local }}^{n(2)}\left(x_{a}, x_{b}\right)=-e_{n} \frac{\eta_{\overline{i j}}}{\left|x_{a}-x_{b}\right|^{2(d-1)}}
$$

for some unfixed coefficient $e_{n}$ depending on the Renyi index $n$. As a function of $n$ we expect that $e_{n}$ is non-universal and theory dependent. We do know that as $n \rightarrow 1$ it should equal $e_{1}=2 \pi^{2} C_{T} /(d+1)$. In $d=3$ we can use this result to make a prediction for the logarithmic term in the Renyi entropies in the presence of a corner. Following the same steps as in section 4.1 we find the coefficient of the log is

$$
a_{n}(\theta)=\frac{e_{n}^{(d=3)}}{12}(\pi-\theta)^{2}+\ldots
$$

for opening angles close to $\pi$. Quite a bit is known about such contributions to the corner terms in Renyi entropies which we may then use to make predictions about $e_{n}$. For example the conjecture in [28] would lead to the relation:

$$
e_{n}=\frac{12 h_{n}}{(n-1) \pi}
$$

where $h_{n}$ is the (higher dimensional) twist operator dimension which can be related to a one-point function of the stress tensor for the CFT living on the space $\mathbb{H}_{d-1} \times S_{(n)}^{1}$ where the radius of the circle $S^{1}$ has been enlarged by a factor of $n$ relative to the conformally flat version. Thus if we could establish (5.1) for the entanglement density then it would prove the conjecture of [28]. Our field theory approach applied to this problem would naively

\footnotetext{
${ }^{15}$ For $2 \mathrm{~d}$ CFTs however this statement has for the most part been established [48].
} 
suggest $e_{n}$ is related to an integrated (connected) stress tensor two-point function for the CFT living on $\mathbb{H}_{d-1} \times S_{(n)}^{1}$ and it is not at all clear (without putting too much thought into it) how this could be related to a one-point function on the same space.

Further generalizations, that we hope to pursue in the future, include the computation of higher order terms in the perturbative expansion of the shape deformation as well as studying entanglement density in relativistic non-conformal theories.

Finally we comment on previous studies of second order shape deformations of EE in [22] using similar CFT techniques (see also [51] for the Renyi entropy case). These authors considered more general metric deformations that contain the shape deformation as a special case and attempt to access the universal logarithmic divergences in EE for $d=4$ - first written down using different arguments in [52]. This should be contrasted with our approach of examining the non-local (finite) shape dependent part of EE. Certain issues with this CFT perturbative approach were identified in [22], which we suspect would be resolved by a more careful analysis of the relative entropy term along the lines in this paper. However it is not clear that these universal $l n$ terms can be extracted using a nonlocal finite term in EE, say deformed by a more general metric, which was the origin of the many simplifications that occurred in our calculation.

\section{Acknowledgments}

We would like to thank Srivatsan Balakrishnan, Ling-Yan Hung, Mark Mezei, Rob Myers, Mukund Rangamani, Vladimir Rosenhaus, Misha Smolkin and Huajia Wang for helpful conversations. TF and OP would like to thank the Kavli Institute for Theoretical Physics, Santa Barbara where some of this work was carried out, for support under the U.S. National Foundation grant number NSF PHY11-25915. Work supported in part by the U.S. Department of Energy contract DE-FG02-13ER42001. TF is supported by the DARPA, YFA Grant No. D15AP00108.

\section{A Perturbative change in the reduced density matrix}

In this appendix, we prove the formula (3.5) for the perturbative expansion of the reduced density matrix on the subregion $A$ in terms of the metric perturbation $\delta g_{\mu \nu}$. Consider a generic relativistic quantum field theory that admits a path-integral description in terms of the field $\phi$, which collectively denotes all the fields over which we integrate. The density matrix corresponding to the ground state wavefunction on the Cauchy surface $\Sigma$ is given by

$$
|0\rangle\langle 0|=\lim _{\beta \rightarrow \infty} \frac{e^{-\beta \widehat{H}}}{\operatorname{tr}_{\mathfrak{h}_{\Sigma}}\left(e^{-\beta \widehat{H}}\right)}
$$

where $\widehat{H}$ is the Hamiltonian, and $\mathfrak{h}_{\Sigma}$ is the entire Hilbert space. In the path integral language, we can describe a matrix element of this density matrix as a product of path integrals over the regions $x_{E}^{0}>0$ and $x_{E}^{0}<0$ of Euclidean space $\mathbb{R}^{d}$ with the appropriate 
boundary conditions

$$
\left\langle\phi_{-} \mid 0\right\rangle\left\langle 0 \mid \phi_{+}\right\rangle=\frac{1}{Z} \int_{\phi\left(0^{-}, \mathbf{x}\right)=\phi_{-}(\mathbf{x})}[D \phi]_{x_{E}^{0}<0} e^{-S[\phi]} \int_{\phi\left(0^{+}, \mathbf{x}\right)=\phi_{+}(\mathbf{x})}[D \phi]_{x_{E}^{0}>0} e^{-S[\phi]}
$$

where we have denoted the spatial coordinates collectively as $\mathbf{x}=\left(x^{1}, x^{i}\right)$, and $Z$ is the partition function of the theory on $\mathbb{R}^{d}$.

Let us now denote the reduced density matrix for the half space $A=\left\{\mathbf{x}_{A}=\right.$ $\left.\left(x^{1}, x^{i}\right) \mid x^{1}>0\right\}$ by $\widehat{\rho}_{0}$. The matrix element $\left\langle\phi_{-}^{A}\left|\widehat{\rho}_{0}\right| \phi_{+}^{A}\right\rangle$ is then given by gluing the above path integrals along the complementary space $\bar{A}$ at $x_{E}^{0}=0$

$$
\left\langle\phi_{-}^{A}\left|\widehat{\rho}_{0}\right| \phi_{+}^{A}\right\rangle=\frac{1}{Z} \int_{\phi\left(0^{+}, \mathbf{x}_{A}\right)=\phi_{+}^{A}}^{\phi\left(0^{-}, \mathbf{x}_{A}\right)=\phi_{-}^{A}}[D \phi] e^{-S[\phi]}
$$

where $\mathbf{x}_{A}$ are spatial coordinates on $A$, and $\phi_{ \pm}^{A}$ denotes a field configuration restricted to $A$. By slicing this path integral along the angular direction $\theta$ in the $\left(x_{E}^{0}, x^{1}\right)$ plane, it becomes immediately clear that the reduced density matrix can be written in operator form as [36]

$$
\widehat{\rho}_{0}=\frac{e^{-2 \pi \widehat{K}}}{\operatorname{tr}_{\mathfrak{h}_{A}}\left(e^{-2 \pi \widehat{K}}\right)}
$$

where $\mathfrak{h}_{A}$ is the Hilbert space on $A$, and $\widehat{K}$ is the generator of $\theta$-rotations

$$
\widehat{K}=\int d^{d-2} x^{i} \int_{0}^{\infty} d x^{1} x^{1} \widehat{T}^{00}\left(0, x^{1}, x^{i}\right) .
$$

From equation (A.4), we see that up to an overall shift coming from the normalization, the entanglement Hamiltonian in this case is given by

$$
\widehat{H}_{E} \equiv-\ln \widehat{\rho}_{0}=2 \pi \widehat{K}+\text { constant. }
$$

Next, we turn on a small (background) metric deformation $\delta g_{\mu \nu}$. The new reduced density matrix $\widehat{\rho}$ is given by

$$
\left\langle\phi_{-}^{A}|\widehat{\rho}| \phi_{+}^{A}\right\rangle=\frac{1}{(Z+\delta Z)} \int_{\phi\left(0^{+}, \mathbf{x}^{A}\right)=\phi_{+}^{A}}^{\phi\left(0^{-}, \mathbf{x}^{A}\right)=\phi_{-}^{A}}[D \phi] e^{-S[\phi]+\frac{1}{2} \int d^{d} \boldsymbol{x} \delta g^{\mu \nu}(\boldsymbol{x}) T_{\mu \nu}(\boldsymbol{x})+\cdots}
$$

where we have introduced $\boldsymbol{x}^{\mu}=\left(x_{E}^{0}, \mathbf{x}\right)$ to collectively denote the coordinates on $\mathbb{R}^{d}$. One might worry about an extra term in the exponential coming from a change in the stress tensor upon introducing $\delta g$. However, at second order in the $\delta g$-expansion, such a term can at best give a local contribution, and so we drop it. Therefore to quadratic order in $\delta g$, we have

$$
\begin{aligned}
\left\langle\phi_{-}^{A}|\delta \widehat{\rho}| \phi_{+}^{A}\right\rangle= & \frac{1}{2 Z} \int_{\phi_{+}^{A}}^{\phi_{-}^{A}}[D \phi] e^{-S[\phi]}\left\{\frac{1}{2} \int d^{d} \boldsymbol{x} \delta g_{\mu \nu}(\boldsymbol{x})\left(T^{\mu \nu}(\boldsymbol{x})-\left\langle T^{\mu \nu}(\boldsymbol{x})\right\rangle\right)\right. \\
& +\frac{1}{8} \int d^{d} \boldsymbol{x}_{a} d^{d} \boldsymbol{x}_{b} \delta g_{\mu \nu}\left(\boldsymbol{x}_{a}\right) \delta g_{\lambda \sigma}\left(\boldsymbol{x}_{b}\right)\left(T^{\mu \nu}\left(\boldsymbol{x}_{a}\right) T^{\lambda \sigma}\left(\boldsymbol{x}_{b}\right)-2 T^{\mu \nu}\left(\boldsymbol{x}_{a}\right)\left\langle T^{\lambda \sigma}\left(\boldsymbol{x}_{b}\right)\right\rangle\right. \\
& \left.\left.-\left\langle T^{\mu \nu}\left(\boldsymbol{x}_{a}\right) T^{\lambda \sigma}\left(\boldsymbol{x}_{b}\right)\right\rangle+2\left\langle T^{\mu \nu}\left(\boldsymbol{x}_{a}\right)\right\rangle\left\langle T^{\lambda \sigma}\left(\boldsymbol{x}_{b}\right)\right\rangle\right)+\cdots\right\} .
\end{aligned}
$$


From equation (A.4), we can then infer the following operator expression for the change in the reduced density matrix

$$
\begin{aligned}
\delta \widehat{\rho}= & \frac{1}{2} \int d^{d} \boldsymbol{x} \delta g_{\mu \nu}(\boldsymbol{x}) \widehat{\rho}_{0}\left(\widehat{T}^{\mu \nu}(\boldsymbol{x})-\operatorname{tr}_{\mathfrak{h}_{A}}\left(\widehat{\rho}_{0} \widehat{T}^{\mu \nu}(\boldsymbol{x})\right)\right) \\
& +\frac{1}{8} \int d^{d} \boldsymbol{x}_{a} d^{d} \boldsymbol{x}_{b} \delta g_{\mu \nu}\left(\boldsymbol{x}_{a}\right) \delta g_{\lambda \sigma}\left(\boldsymbol{x}_{b}\right) \widehat{\rho}_{0}\left\{\mathcal{T}\left[\widehat{T}^{\mu \nu}\left(\boldsymbol{x}_{a}\right) \widehat{T}^{\lambda \sigma}\left(\boldsymbol{x}_{b}\right)\right]-2 \widehat{T}^{\mu \nu}\left(\boldsymbol{x}_{a}\right) \operatorname{tr}_{\mathfrak{h}_{A}}\left(\widehat{\rho}_{0} \widehat{T}^{\lambda \sigma}\left(\boldsymbol{x}_{b}\right)\right)\right. \\
& \left.-\operatorname{tr}_{\mathfrak{h}_{A}}\left(\widehat{\rho}_{0} \mathcal{T}\left[\widehat{T}^{\mu \nu}\left(\boldsymbol{x}_{a}\right) \widehat{T}^{\lambda \sigma}\left(\boldsymbol{x}_{b}\right)\right]\right)+2 \operatorname{tr}_{\mathfrak{h}_{A}}\left(\widehat{\rho}_{0} \widehat{T}^{\mu \nu}\left(\boldsymbol{x}_{a}\right)\right) \operatorname{tr}_{\mathfrak{h}_{A}}\left(\widehat{\rho}_{0} \widehat{T}^{\lambda \sigma}\left(\boldsymbol{x}_{b}\right)\right)\right\}+\cdots .(\mathrm{A} .11)
\end{aligned}
$$

Switching to polar coordinates $(r, \theta)$ in the $\left(x_{0}^{E}, x^{1}\right)$ plane, the operator $\widehat{T}\left(\theta, r, x^{i}\right)$ above is to be interpreted (from the point of view of the reduced density matrix) as

$$
\widehat{T}^{\mu \nu}\left(\theta, r, x^{i}\right)=(R(\theta))_{\lambda}^{\mu}(R(\theta))_{\sigma}^{\nu} e^{\theta \widehat{K}^{T}} \widehat{T}^{\lambda \sigma}\left(0, r, x^{i}\right) e^{-\theta \widehat{K}}
$$

where $R(\theta)$ is the appropriate rotation matrix in the vector representation. Further, $\mathcal{T}$ is the angular-ordering operator in the $\left(x_{E}^{0}, x^{1}\right)$ plane

$$
\mathcal{T}\left[\widehat{\mathcal{O}}\left(\theta_{a}\right) \widehat{\mathcal{O}}\left(\theta_{b}\right)\right]=\widehat{\mathcal{O}}\left(\theta_{a}\right) \widehat{\mathcal{O}}\left(\theta_{b}\right) H\left(\theta_{a}-\theta_{b}\right)+\widehat{\mathcal{O}}\left(\theta_{b}\right) \widehat{\mathcal{O}}\left(\theta_{a}\right) H\left(\theta_{b}-\theta_{a}\right)
$$

where $H\left(\theta_{a}-\theta_{b}\right)$ is the Heaviside step function.

\section{B Angular ordering in the relative entropy term}

Recall that the relative entropy term is given by

$$
\begin{aligned}
\delta S_{E E}^{(2)}= & -\frac{1}{4} \int d^{d} \boldsymbol{x}_{a} d^{d} \boldsymbol{x}_{b} \delta g_{\mu \nu}\left(\boldsymbol{x}_{a}\right) \delta g_{\lambda \sigma}\left(\boldsymbol{x}_{b}\right) \\
& \times \int_{0}^{\infty} d \beta \beta \operatorname{tr}_{\mathfrak{h}_{A}}\left(\frac{\widehat{\rho}_{0}}{\left(\widehat{\rho}_{0}+\beta\right)^{2}}: \widehat{T}^{\mu \nu}:\left(\boldsymbol{x}_{a}\right) \frac{\widehat{\rho}_{0}}{\widehat{\rho}_{0}+\beta}: \widehat{T}^{\lambda \sigma}:\left(\boldsymbol{x}_{b}\right)\right)
\end{aligned}
$$

where we have used the short notation

$$
: \widehat{T}^{\mu \nu}:\left(\boldsymbol{x}_{a}\right)=\widehat{T}^{\mu \nu}\left(\boldsymbol{x}_{a}\right)-\operatorname{tr}_{\mathfrak{h}_{A}}\left(\widehat{\rho}_{0} \widehat{T}^{\mu \nu}\left(\boldsymbol{x}_{a}\right)\right)
$$

Unfortunately, the above expression is not $\mathcal{T}$-ordered, and cannot be written in terms of a Euclidean correlation function. To resolve this problem, we will perform the $\beta$-integral, and then manipulate the expression further to bring it into a $\mathcal{T}$-ordered form. Let's begin by rewriting it as

$$
\begin{aligned}
& \delta S_{E E}^{(2)}=-\frac{1}{4} \int d^{d} \boldsymbol{x}_{a} d^{d} \boldsymbol{x}_{b} \widetilde{\delta g_{\mu \nu}}\left(\boldsymbol{x}_{a}\right) \widetilde{\delta g}_{\lambda \sigma}\left(\boldsymbol{x}_{b}\right) \\
& \quad \times \int_{0}^{\infty} d \beta \beta \operatorname{tr}_{\mathfrak{h}_{A}}\left(\frac{\widehat{\rho}_{0}}{\left(\widehat{\rho}_{0}+\beta\right)^{2}} e^{\theta_{a} \widehat{K}}: \widehat{T}^{\mu \nu}:\left(0, r_{a}, x_{a}^{i}\right) e^{-\theta_{a} \widehat{K}} \frac{\widehat{\rho}_{0}}{\widehat{\rho}_{0}+\beta} e^{\theta_{b} \widehat{K}}: \widehat{T}^{\lambda \sigma}:\left(0, r_{b}, x_{b}^{i}\right) e^{-\theta_{b} \widehat{K}}\right)
\end{aligned}
$$

where we have defined

$$
\widetilde{\delta g}_{\mu \nu}(\boldsymbol{x})=\delta g_{\lambda \sigma}(\boldsymbol{x})(R(\theta))_{\mu}^{\lambda}(R(\theta))_{\nu}^{\sigma}
$$


Let us denote the first line of (B.3) as $-\int d \tilde{\mu}_{\mu \nu \lambda \sigma}$ for convenience. Using the eigenstates of $\widehat{K}$ defined by $\widehat{K}|\omega\rangle=\omega|\omega\rangle$ to carry out the above trace, and writing $\widehat{\rho}_{0}=c e^{-2 \pi \widehat{K}}$, we get

$$
\begin{aligned}
\delta S_{E E}^{(2)}= & -\int d \tilde{\mu}_{\mu \nu \lambda \sigma} \sum_{\omega_{a}, \omega_{b}} e^{\left(\theta_{a}-\theta_{b}\right)\left(\omega_{a}-\omega_{b}\right)}\left\langle\omega_{a}\left|: \widehat{T}^{\mu \nu}:\left(0, r_{a}, x_{a}^{i}\right)\right| \omega_{b}\right\rangle\left\langle\omega_{b}\left|: \widehat{T}^{\lambda \sigma}:\left(0, r_{b}, x_{b}^{i}\right)\right| \omega_{a}\right\rangle \\
& \times c \int_{0}^{\infty} d \beta \beta \frac{e^{-2 \pi\left(\omega_{a}+\omega_{b}\right)}}{\left(e^{-2 \pi \omega_{a}}+\beta\right)^{2}\left(\beta+e^{\left.-2 \pi \omega_{b}\right)}\right.} .
\end{aligned}
$$

The $\beta$ integral can be performed to obtain

$$
\int_{0}^{\infty} d \beta \beta \frac{e^{-2 \pi\left(\omega_{a}+\omega_{b}\right)}}{\left(e^{-2 \pi \omega_{a}}+\beta\right)^{2}\left(\beta+e^{\left.-2 \pi \omega_{b}\right)}\right.}=e^{-2 \pi \omega_{b}}\left(\frac{\nu e^{\nu}}{\left(1-e^{\nu}\right)^{2}}+\frac{1}{1-e^{\nu}}\right)
$$

where $\nu=2 \pi\left(\omega_{a}-\omega_{b}\right)$. Next, using the formulae

$$
\begin{aligned}
\left(\frac{1}{1-e^{\nu}}+\frac{\nu e^{\nu}}{\left(1-e^{\nu}\right)^{2}}\right) & =\int_{-\infty-i \varepsilon}^{\infty-i \varepsilon} \frac{d s}{2 \pi i} e^{-i \nu s / 2 \pi} \frac{s}{4 \sinh ^{2}(s / 2)} \\
\left(\frac{1}{1-e^{\nu}}+\frac{\nu e^{\nu}}{\left(1-e^{\nu}\right)^{2}}\right) & =\int_{-\infty+i \varepsilon}^{\infty+i \varepsilon} \frac{d s}{2 \pi i} e^{-i \nu s / 2 \pi-\nu} \frac{s-2 \pi i}{4 \sinh ^{2}(s / 2)}
\end{aligned}
$$

allows us to revert back from the spectral representation to the operator-trace form. To proceed, let's split the integral in eq (B.5) into two parts: $\theta_{a}>\theta_{b}$ and $\theta_{b}>\theta_{a}$. For the first integral, we use equation (B.8) and for the second integral we use (B.7)

$$
\begin{aligned}
\delta S_{E E, \theta_{a}>\theta_{b}}^{(2)}= & \int_{\theta_{a}>\theta_{b}} d \mu_{\mu \nu \lambda \sigma} \int_{\mathbb{R}+i \varepsilon} \frac{d s}{2 \pi i} \frac{2 \pi i-s}{4 \sinh ^{2}(s / 2)} \\
& \times\left(R^{-1}(i s)\right)^{\lambda}{ }_{\kappa}\left(R^{-1}(i s)\right)^{\sigma}{ }_{\eta} \operatorname{tr}_{\mathfrak{h}_{A}, \text { conn. }}\left(\widehat{\rho}_{0} \widehat{T}^{\mu \nu}\left(\theta_{a}, r_{a}, x_{a}^{i}\right) \widehat{T}^{\kappa \eta}\left(\theta_{b}+i s, r_{b}, x_{b}^{i}\right)\right) \\
\delta S_{E E, \theta_{a}<\theta_{b}}^{(2)}= & -\int_{\theta_{a}<\theta_{b}} d \mu_{\mu \nu \lambda \sigma} \int_{\mathbb{R}-i \varepsilon} \frac{d s}{2 \pi i} \frac{s}{4 \sinh ^{2}(s / 2)} \\
& \times\left(R^{-1}(i s)\right)^{\lambda}{ }_{\kappa}\left(R^{-1}(i s)\right)^{\sigma}{ }_{\eta} \operatorname{tr}_{\mathfrak{h}_{A}, \text { conn. }}\left(\widehat{\rho}_{0} \widehat{T}^{\kappa \eta}\left(\theta_{b}+i s, r_{b}, x_{b}^{i}\right) \widehat{T}^{\mu \nu}\left(\theta_{a}, r_{a}, x_{a}^{i}\right)\right)
\end{aligned}
$$

where $\int d \mu_{\mu \nu \lambda \sigma}=\frac{1}{4} \int d^{d} \boldsymbol{x}_{a} d^{d} \boldsymbol{x}_{b} \delta g_{\mu \nu}\left(\boldsymbol{x}_{a}\right) \delta g_{\lambda \sigma}\left(\boldsymbol{x}_{b}\right)$, and we have introduced the connected trace

$$
\operatorname{tr}_{\mathfrak{h}_{A}, \text { conn. }}\left(\widehat{\rho}_{0} \widehat{A} \cdot \widehat{B}\right)=\operatorname{tr}_{\mathfrak{h}_{A}}\left(\widehat{\rho}_{0} \widehat{A} \cdot \widehat{B}\right)-\operatorname{tr}_{\mathfrak{h}_{A}}\left(\widehat{\rho}_{0} \widehat{A}\right) \operatorname{tr}_{\mathfrak{h}_{A}}\left(\widehat{\rho}_{0} \widehat{B}\right)
$$

Now making the replacements $\boldsymbol{x}_{a} \leftrightarrow \boldsymbol{x}_{b}$ and $s \rightarrow-s$ in (B.10), and adding (B.9) and (B.10), we obtain

$$
\begin{aligned}
\delta S_{E E}^{(2)}= & \int_{\theta_{a}>\theta_{b}} d \mu_{\mu \nu \lambda \sigma} \int_{+i \varepsilon} \frac{d s}{4 \sinh ^{2}(s / 2)} \\
& \times\left(R^{-1}(i s)\right)^{\lambda}{ }_{\kappa}\left(R^{-1}(i s)\right)^{\sigma}{ }_{\eta} \operatorname{tr}_{\mathfrak{h}_{A}, \text { conn. }}\left(\widehat{\rho}_{0} \widehat{T}^{\mu \nu}\left(\theta_{a}, r_{a}, x_{a}^{i}\right) \widehat{T}^{\kappa \eta}\left(\theta_{b}+i s, r_{b}, x_{b}^{i}\right)\right) .
\end{aligned}
$$

Finally, once again making the replacements $\boldsymbol{x}_{a} \leftrightarrow \boldsymbol{x}_{b}$ and $s \rightarrow-s$ in the above integral, and adding to itself, we obtain

$$
\begin{aligned}
\delta S_{E E}^{(2)}= & \frac{1}{8} \int d^{d} \boldsymbol{x}_{a} d^{d} \boldsymbol{x}_{b} \delta g_{\mu \nu}\left(\boldsymbol{x}_{a}\right) \delta g_{\lambda \sigma}\left(\boldsymbol{x}_{b}\right) \int_{C} \frac{d s}{4 \sinh ^{2}(s / 2)}\left(R^{-1}(i s)\right)^{\lambda}\left(R^{-1}(i s)\right)^{\sigma}{ }_{\eta} \operatorname{tr}_{\mathfrak{h}_{A}, \text { conn. }} \\
& \times\left(\widehat{\rho}_{0} \mathcal{T}\left[\widehat{T}^{\mu \nu}\left(\theta_{a}, r_{a}, x_{a}^{i}\right) \widehat{T}^{\kappa \eta}\left(\theta_{b}+i s, r_{b}, x_{b}^{i}\right)\right]\right)
\end{aligned}
$$

where the contour is given by $C=\mathbb{R}+i \varepsilon \operatorname{sign}\left(\theta_{a}-\theta_{b}\right)$. This then gives the result (3.16) used in the main text. 


\section{Contact \& crossing terms}

In this appendix, we analyse (i) the contact terms in equation (3.50), (ii) crossing terms in the three-point function term in (3.50).

\section{C.1 Contact terms}

The contact terms in (3.50) are given by

$$
\begin{aligned}
\text { contact terms }= & \int_{\mathcal{H}_{\Lambda}} \int_{\partial \mathcal{H}_{\Lambda}} \xi_{\beta}\left(y_{a}\right) n_{\gamma}\left(y_{b}\right) \xi_{\delta}\left(y_{b}\right) \nabla_{\alpha}^{(a)}\left\langle\widehat{T}^{\alpha \beta}\left(y_{a}\right) \widehat{T}^{\gamma \delta}\left(y_{b}\right) \widehat{H}_{E}\right\rangle \\
& +\frac{1}{2} \int_{\mathcal{H}_{\Lambda}} \int_{\mathcal{H}_{\Lambda}} \xi_{\beta}\left(y_{a}\right) \xi_{\delta}\left(y_{b}\right) \nabla_{\alpha}^{(a)} \nabla_{\gamma}^{(b)}\left\langle\widehat{T}^{\alpha \beta}\left(y_{a}\right) \widehat{T}^{\gamma \delta}\left(y_{b}\right) \widehat{H}_{E}\right\rangle \\
& +\frac{1}{2} \int_{\partial \mathcal{H}_{\Lambda}} \int_{\mathcal{H}_{\Lambda}} n_{\alpha}\left(y_{a}\right) \xi_{\beta}\left(y_{a}\right) \Xi\left(y_{b}\right)\left\langle\widehat{T}^{\alpha \beta}\left(y_{a}\right) \widehat{T}_{\gamma}^{\gamma}\left(y_{b}\right) \widehat{H}_{E}\right\rangle \\
& -\frac{1}{2} \int_{\mathcal{H}_{\Lambda}} \int_{\mathcal{H}_{\Lambda}} \xi_{\beta}\left(y_{a}\right) \Xi\left(y_{b}\right) \nabla_{\alpha}^{(a)}\left\langle\widehat{T}^{\alpha \beta}\left(y_{a}\right) \widehat{T}_{\gamma}^{\gamma}\left(y_{b}\right) \widehat{H}_{E}\right\rangle \\
& +\frac{1}{8} \int_{\mathcal{H}_{\Lambda}} \int_{\mathcal{H}_{\Lambda}} \Xi\left(y_{a}\right) \Xi\left(y_{b}\right)\left\langle\widehat{T}_{\alpha}^{\alpha}\left(y_{a}\right) \widehat{T}_{\gamma}^{\gamma}\left(y_{b}\right) \widehat{H}_{E}\right\rangle
\end{aligned}
$$

where we have defined $\Xi=\xi^{\alpha} \partial_{\alpha} \ln \Omega^{2}$. The modular Hamiltonian can be written as the following integral over the constant $\tau=\tau_{c}$ slice

$$
\widehat{H}_{E}=2 \pi \int_{\mathbb{H}^{d-1}} \frac{d^{d-2} x_{c}^{i} d z_{c}}{z_{c}^{d-1}} \widehat{T}_{\tau \tau}\left(\tau_{c}, z_{c}, x_{c}^{i}\right)+\text { constant. }
$$

We will need to use the diffeomorphism and trace Ward identities for three-point functions of stress tensors, which can be found in [41] (for Euclidean space). On a general manifold with the metric $g_{\mu \nu}$, these Ward identities take the following form

$$
\begin{aligned}
& \nabla_{(x)}^{\mu}\left\langle\widehat{T}_{\mu \nu}(x) \widehat{T}_{\lambda \sigma}(y) \widehat{T}_{\rho \kappa}(z)\right\rangle=\nabla_{\nu}^{(x)}\left(\frac{\delta^{d}(x-y)}{\sqrt{g}}\right)\left\langle\widehat{T}_{\lambda \sigma}(x) \widehat{T}_{\rho \kappa}(z)\right\rangle \\
& +2 \nabla_{(\lambda}^{(x)}\left(\frac{\delta^{d}(x-y)}{\sqrt{g}}\left\langle\widehat{T}_{\sigma) \nu}(x) \widehat{T}_{\rho \kappa}(z)\right\rangle\right) \\
& +\nabla_{\nu}^{(x)}\left(\frac{\delta^{d}(x-z)}{\sqrt{g}}\right)\left\langle\widehat{T}_{\lambda \sigma}(y) \widehat{T}_{\rho \kappa}(x)\right\rangle \\
& +2 \nabla_{(\rho}^{(x)}\left(\frac{\delta^{d}(x-z)}{\sqrt{g}}\left\langle\widehat{T}_{\kappa) \nu}(x) \widehat{T}_{\lambda \sigma}(y)\right\rangle\right) \\
& g^{\mu \nu}(x)\left\langle\widehat{T}_{\mu \nu}(x) \widehat{T}_{\lambda \sigma}(y) \widehat{T}_{\rho \kappa}(z)\right\rangle=2\left(\frac{\delta^{d}(x-y)}{\sqrt{g}}+\frac{\delta^{d}(x-z)}{\sqrt{g}}\right)\left\langle\widehat{T}_{\lambda \sigma}(y) \widehat{T}_{\rho \kappa}(z)\right\rangle .
\end{aligned}
$$

Using these identities, we see that most of the contact terms in (C.1) drop out trivially because $y_{a}$ and $y_{b}$ are well separated (i.e., because we are only keeping terms which contribute to the non-local part of the entanglement density). The only potentially non-trivial terms are

$$
\begin{aligned}
\text { contact terms }= & -\int_{\mathcal{H}_{\Lambda}} \int_{\partial \mathcal{H}_{\Lambda}} \xi_{\beta}\left(y_{a}\right) n_{\gamma}\left(y_{b}\right) \xi_{\delta}\left(y_{b}\right) \nabla_{\alpha}^{(a)}\left\langle\widehat{T}^{\alpha \beta}\left(y_{a}\right) \widehat{T}^{\gamma \delta}\left(y_{b}\right) \widehat{H}_{E}\right\rangle \\
& +\frac{1}{2} \int_{\partial \mathcal{H}_{\Lambda}} \int_{\mathcal{H}_{\Lambda}} n_{\alpha}\left(y_{a}\right) \xi_{\beta}\left(y_{a}\right) \Xi\left(y_{b}\right)\left\langle\widehat{T}^{\alpha \beta}\left(y_{a}\right) \widehat{T}_{\gamma}^{\gamma}\left(y_{b}\right) \widehat{H}_{E}\right\rangle .
\end{aligned}
$$


Before proceeding to consider these terms, we first pause to observe that both the above terms are independent of the time coordinate $\tau_{c}$ at which the modular Hamiltonian is placed. This is because $\widehat{H}_{E}$ is a conserved charge, and we can freely move it in $\tau_{c}$ as long as we don't cross other operators. When we do in fact cross another operator, say $\widehat{T}^{\alpha \beta}\left(y_{a}\right)$, then using the commutator $\left[\widehat{H}_{E}, \widehat{T}^{\alpha \beta}\left(y_{a}\right)\right] \sim \partial_{\tau_{a}} \widehat{T}^{\alpha \beta}\left(y_{a}\right)$, we generate extra terms involving the two-point functions of stress tensors. However, using the Ward identities for the 2-point functions, it is straightforward to check that such crossing terms in (C.5) vanish for $y_{a}$ and $y_{b}$ well-separated. Thus, we conclude that both the terms in (C.5) are independent of $\tau_{c}$.

In light of the above discussion, we can simplify the integrals in (C.5) by integrating over $\tau_{c}$ (and dividing by $2 \pi$ ). For instance, the first term in (C.5) upon using the diffeomorphism Ward identity and integrating over $\tau_{c}$ gives

$$
\begin{aligned}
1 s t \text { term }= & -\frac{1}{2 \pi} \int_{\mathcal{H}_{\Lambda}} d \mu_{a} \int_{\partial \mathcal{H}_{\Lambda}} d \bar{\mu}_{b} \int_{\mathcal{H}_{\Lambda}} d \mu_{c} \xi^{\beta}\left(y_{a}\right) n^{\gamma}\left(y_{b}\right) \xi^{\delta}\left(y_{b}\right) \\
& \times\left[\nabla_{\beta}^{(a)}\left(\frac{\delta\left(y_{a}-y_{c}\right)}{\sqrt{g^{\mathcal{H}}}}\right)\left\langle\widehat{T}_{\gamma \delta}\left(y_{b}\right) \widehat{T}_{\tau \tau}\left(y_{a}\right)\right\rangle+2 \partial_{\tau}^{(a)}\left(\frac{\delta\left(y_{a}-y_{c}\right)}{\sqrt{g^{\mathcal{H}}}}\left\langle\widehat{T}_{\beta \tau}\left(y_{a}\right) \widehat{T}_{\gamma \delta}\left(y_{b}\right)\right\rangle\right)\right] \\
= & \frac{1}{\pi} \int_{\mathcal{H}_{\Lambda}} d \mu_{a} \int_{\partial \mathcal{H}_{\Lambda}} d \bar{\mu}_{b} \partial_{\tau}^{(a)} \xi^{\beta}\left(y_{a}\right) n^{\gamma}\left(y_{b}\right) \xi^{\delta}\left(y_{b}\right)\left\langle\widehat{T}_{\beta \tau}\left(y_{a}\right) \widehat{T}_{\gamma \delta}\left(y_{b}\right)\right\rangle
\end{aligned}
$$

where in the second line we have performed the $y_{c}$ integration, and once again we have taken $y_{a}$ and $y_{b}$ to be well-separated. The two-point function appearing above can be computed efficiently using the embedding space formalism. Having done so, one finds that the above term is suppressed by a factor of $\frac{1}{\Lambda}$. The only thing to check is whether the $z_{a}$ integral inside $d \mu_{a}$ is divergent, because such divergences could give potential enhancements. Happily, one finds that the $z_{a}$ integral is finite, and thus the above term vanishes in the limit $\Lambda \rightarrow \infty$. Similarly, one can check that the second term in (C.5) also vanishes as $\Lambda \rightarrow \infty$.

\section{C.2 Crossing terms}

Next, we argue that the three-point function term in (3.50)

$$
\delta S_{E E}^{(1)}=\frac{1}{8} \int_{\partial \mathcal{H}_{\Lambda}} d \bar{\mu}_{a} n^{\alpha}\left(y_{a}\right) \xi^{\beta}\left(y_{a}\right) \int_{\partial \mathcal{H}_{\Lambda}} d \bar{\mu}_{b} n^{\gamma}\left(y_{b}\right) \xi^{\delta}\left(y_{b}\right)\left\langle\widehat{T}_{\alpha \beta}\left(y_{a}\right) \widehat{T}_{\gamma \delta}\left(y_{b}\right) \widehat{H}_{E}\right\rangle_{\mathcal{H}}
$$

is independent of the time $\tau_{c}$ at which we place the modular Hamiltonian. Since the modular Hamiltonian is a conserved charge, we are indeed free to move it around in $\tau$, as long as we don't cross another operator insertion. However, when we do cross another operator, we pick up an extra contact (or commutator) term, which we will refer to as a crossing term. For instance, let us take $\tau_{c}$ from $\tau_{a}-\epsilon$ to $\tau_{a}+\epsilon$; in this case we pick up the crossing term

$$
\begin{aligned}
& =\frac{1}{8} \int_{\partial \mathcal{H}_{\Lambda}} d \bar{\mu}_{a} n^{\alpha}\left(y_{a}\right) \xi^{\beta}\left(y_{a}\right) \int_{\partial \mathcal{H}_{\Lambda}} d \bar{\mu}_{b} n^{\gamma}\left(y_{b}\right) \xi^{\delta}\left(y_{b}\right)\left\langle\left[\widehat{H}_{E}, \widehat{T}_{\alpha \beta}\left(y_{a}\right)\right] \widehat{T}_{\gamma \delta}\left(y_{b}\right)\right\rangle_{\mathcal{H}} \\
& =\frac{1}{8} \int_{\partial \mathcal{H}_{\Lambda}} d \bar{\mu}_{a} n^{\alpha}\left(y_{a}\right) \xi^{\beta}\left(y_{a}\right) \int_{\partial \mathcal{H}_{\Lambda}} d \bar{\mu}_{b} n^{\gamma}\left(y_{b}\right) \xi^{\delta}\left(y_{b}\right) \partial_{\tau_{a}}\left\langle\widehat{T}_{\alpha \beta}\left(y_{a}\right) \widehat{T}_{\gamma \delta}\left(y_{b}\right)\right\rangle_{\mathcal{H}}
\end{aligned}
$$


Similar to our previous discussion, the two-point function appearing above can be computed using the embedding space formalism. Having done so, one finds that the above term is suppressed by a factor of $\frac{1}{\Lambda^{2}}$. There are no other enhancements to cancel this factor, and the above term simply vanishes in the limit $\Lambda \rightarrow \infty$. Therefore in this limit, the crossing terms can be ignored.

\section{Integral}

In this section, we wish to evaluate the generic integral

$I\left(n_{+}, n_{-}, n_{0} \mid m_{a}, m_{b}\right)=\int_{\mathbb{H}^{d-1}} d Y_{c}\left(-2 P_{a} \cdot P_{c}\right)^{-m_{a}}\left(-2 P_{b} \cdot P_{c}\right)^{-m_{b}}\left(E_{+} \cdot P_{c}\right)^{n_{+}}\left(E_{-} \cdot P_{c}\right)^{n_{-}}\left(E_{0} \cdot P_{c}\right)^{n_{0}}$

which appears in the calculation of the modular Hamiltonian term, in the limit where we send the modular Hamiltonian to the Rindler horizon. Using Schwinger parameters, we can rewrite this integral as

$$
\begin{aligned}
I= & \int_{0}^{\infty} \frac{1}{\Gamma\left(m_{a}\right)} d t_{a} t_{a}^{m_{a}-1} \int_{0}^{\infty} \frac{1}{\Gamma\left(m_{b}\right)} d t_{b} t_{b}^{m_{b}-1} \\
& \times \int_{\mathbb{H}^{d-1}} d Y_{c} e^{2\left(t_{a} P_{a}+t_{b} P_{b}\right) \cdot P_{c}}\left(E_{+} \cdot P_{c}\right)^{n_{+}}\left(E_{-} \cdot P_{c}\right)^{n_{-}}\left(E_{0} \cdot P_{c}\right)^{n_{0}}
\end{aligned}
$$

We will use embedding space coordinates $Y=\left(\frac{1+z^{2}+\left(x^{i}\right)^{2}}{2 z}, \frac{1-z^{2}-\left(x^{i}\right)^{2}}{2 z}, \frac{x^{i}}{z}\right)$ on $\mathbb{H}^{d-1}$. Analytically continuing the above integral in the $w=e^{i \tau_{c}}$ plane and sending $\tau_{c} \rightarrow \mp i \infty$, we find

$$
\begin{aligned}
I= & \beta_{c}^{\left(n_{+}-n_{-}\right)} \int_{0}^{\infty} \frac{1}{\Gamma\left(m_{a}\right)} d t_{a} t_{a}^{m_{a}-1} \int_{0}^{\infty} \frac{1}{\Gamma\left(m_{b}\right)} d t_{b} t_{b}^{m_{b}-1} \int_{\mathbb{H}^{d-1}} d Y_{c}\left(E_{0} \cdot P_{c}\right)^{n_{0}} \\
& \times \exp \left(\left(t_{a} e^{\mp i \tau_{a}}+t_{b} e^{\mp i \tau_{b}}\right) \beta_{c}+2\left(t_{a} Y_{a}+t_{b} Y_{b}\right) \cdot Y_{c}+O\left(\beta_{c}^{-1}\right)\right)
\end{aligned}
$$

where $\beta_{c}=e^{\left|\tau_{c}\right|}$. Now we partition $n_{0}$ into two integers $\alpha+\beta=n_{0}$, and rewrite the above integral as

$$
\begin{aligned}
I= & \frac{\beta_{c}^{\left(n_{+}-n_{-}\right)}}{2^{n_{0}}}\left(E_{0} \cdot \frac{\partial}{\partial Y_{a}}\right)^{\alpha}\left(E_{0} \cdot \frac{\partial}{\partial Y_{b}}\right)^{\beta} \\
& \times \int_{0}^{\infty} \frac{1}{\Gamma\left(m_{a}\right)} d t_{a} t_{a}^{m_{a}-\alpha-1} \int_{0}^{\infty} \frac{1}{\Gamma\left(m_{b}\right)} d t_{b} t_{b}^{m_{b}-\beta-1} \int \frac{d^{d-2} x^{i} d z}{z^{d-1}} e^{\beta_{c}\left(t_{a} P_{a}^{\mp}+t_{b} P_{b}^{\mp}\right)-|W| \frac{1+z^{2}+\vec{x}^{2}}{z}}
\end{aligned}
$$

where in the last line we have rotated $Y_{c}$ to align $W=t_{a} Y_{a}+t_{b} Y_{b}$ with $|W|(1,0, \cdots, 0)$. We have also defined

$$
P_{a, b}^{ \pm}=P_{a, b} \cdot E^{ \pm}
$$


Then, with the change of variables $z=z^{\prime} /|W|, x=x^{\prime} /|W|$, the integral becomes (dropping the primes)

$$
\begin{aligned}
I= & \frac{\beta_{c}^{\left(n_{+}-n_{-}\right)}}{2^{n_{0}}}\left(E_{0} \cdot \frac{\partial}{\partial Y_{a}}\right)^{\alpha}\left(E_{0} \cdot \frac{\partial}{\partial Y_{b}}\right)^{\beta} \int_{0}^{\infty} \frac{1}{\Gamma\left(m_{a}\right)} d t_{a} t_{a}^{m_{a}-\alpha-1} \int_{0}^{\infty} \frac{1}{\Gamma\left(m_{b}\right)} d t_{b} t_{b}^{m_{b}-\beta-1} \\
& \times e^{\beta_{c}\left(t_{a} P_{a}^{\mp}+t_{b} P_{b}^{\mp}\right)} \int \frac{d^{d-2} x^{i} d z}{z^{d-1}} e^{-\frac{|W|^{2}}{z}-\frac{z^{2}+\vec{x}^{2}}{z}} \\
= & \frac{\beta_{c}^{\left(n_{+}-n_{-}\right)}}{2^{n_{0}}}\left(E_{0} \cdot \frac{\partial}{\partial Y_{a}}\right)^{\alpha}\left(E_{0} \cdot \frac{\partial}{\partial Y_{b}}\right)^{\beta} \mathcal{I} .
\end{aligned}
$$

Let us focus on $\mathcal{I}$ for the moment. We now change the order of the $\left(t_{a}, t_{b}\right)$ and $\left(z, x^{i}\right)$ integrals, and rescale $t_{a, b}=\sqrt{z} t_{a, b}^{\prime}$

$$
\begin{aligned}
\mathcal{I}= & \int \frac{d^{d-2} x^{i} d z}{z^{d-1}} z^{\left(m_{a}+m_{b}-n_{0}\right) / 2} e^{-\frac{z^{2}+\vec{x}^{2}}{z}} \\
& \times \int_{0}^{\infty} \frac{1}{\Gamma\left(m_{a}\right)} d t_{a} t_{a}^{m_{a}-\alpha-1} \int_{0}^{\infty} \frac{1}{\Gamma\left(m_{b}\right)} d t_{b} t_{b}^{m_{b}-\beta-1} e^{\sqrt{z} \beta_{c}\left(t_{a} P_{a}^{\mp}+t_{b} P_{a}^{\mp}\right)} e^{W^{2}}
\end{aligned}
$$

Finally, performing the $x^{i}$ integrals, and redefining $z=t_{c}^{2}$, we get

$$
\mathcal{I}=C \int_{0}^{\infty} \int_{0}^{\infty} \int_{0}^{\infty} d^{3} t t_{a}^{m_{a}-1} t_{b}^{m_{b}-1} t_{c}^{m_{c}-1} e^{-\sum_{i, j} t_{i} A_{i j} t_{j}}
$$

where

$$
m_{c}=\left[m_{a}+m_{b}-n_{0}-(d-2)\right]=\left(n_{+}+n_{-}+2\right), \quad C=\frac{2 \pi^{\frac{d-2}{2}}}{\Gamma\left(m_{a}\right) \Gamma\left(m_{b}\right)}
$$

and the matrix $A$ is given by

$$
A_{i j ; \pm}=\left(\begin{array}{ccc}
1 & -Y_{a} \cdot Y_{b} & \frac{\beta_{c}}{2_{c}} P_{a}^{\mp} \\
-Y_{a} \cdot Y_{b} & 1 & \frac{\beta_{c}}{2} P_{b}^{\mp} \\
\frac{\beta_{c}}{2} P_{a}^{\mp} & \frac{\beta_{c}}{2} P_{b}^{\mp} & 1
\end{array}\right)
$$

Rescaling $t_{c} \rightarrow \beta_{c}^{-1} t_{c}$, we obtain

$$
\begin{aligned}
\mathcal{I}= & \beta_{c}^{-m_{c}} C \int_{0}^{\infty} d t_{a} d t_{b} d t_{c} t_{a}^{m_{a}-\alpha-1} t_{b}^{m_{b}-\beta-1} t_{c}^{m_{c}-1} \\
& \times \exp \left(-t_{a}^{2}-t_{b}^{2}-\frac{t_{c}^{2}}{\beta_{c}^{2}}+\left(t_{a} P_{a}^{\mp}+t_{b} P_{b}^{\mp}\right) t_{c}-t_{a} t_{b}\left(-2 Y_{a} \cdot Y_{b}\right)\right) .
\end{aligned}
$$

We can perform the $t_{c}$ integral in the limit $\beta_{c} \rightarrow \infty$. The integral converges for $\tau_{a}, \tau_{b}$ in a neighborhood of $\pi$, and we can then continue the expression outside this region:

$$
\mathcal{I}=\beta_{c}^{-m_{c}} C \Gamma\left(m_{c}\right) \int_{0}^{\infty} d t_{a} d t_{b} t_{a}^{m_{a}-\alpha-1} t_{b}^{m_{b}-\beta-1} \frac{1}{\left(-t_{a} P_{a}^{\mp}-t_{b} P_{b}^{\mp}\right)^{m_{c}}} \exp \left(-t_{a}^{2}-t_{b}^{2}-t_{a} t_{b}\left(-2 Y_{a} \cdot Y_{b}\right)\right) .
$$

Finally switching to new integration variables

$$
t_{a}=\sigma \lambda, \quad t_{b}=\frac{\sigma}{\lambda}
$$


and performing the $\sigma$ integration, we obtain

$$
\begin{aligned}
\mathcal{I}= & -\beta_{c}^{-m_{c}} C \Gamma\left(m_{c}\right) \Gamma\left(\frac{d-2}{2}\right) \\
& \times \int_{0}^{\infty} d \lambda \lambda^{m_{a}-\alpha-m_{b}+\beta-1} \frac{1}{\left(-\lambda P_{a}^{\mp}-\lambda^{-1} P_{b}^{\mp}\right)^{m_{c}}} \frac{1}{\left(\lambda^{2}+\frac{1}{\lambda^{2}}-2 Y_{a} \cdot Y_{b}\right)^{\frac{d-2}{2}}} .
\end{aligned}
$$

So the full integral becomes

$$
\begin{gathered}
I\left(n_{+}, n_{-}, n_{0} \mid m_{a}, m_{b}\right)=-\frac{\beta_{c}^{-2-2 n_{-}}}{2^{n_{0}}} C \Gamma\left(m_{c}\right) \Gamma\left(\frac{d-2}{2}\right)\left(E_{0} \cdot \frac{\partial}{\partial Y_{a}}\right)^{\alpha}\left(E_{0} \cdot \frac{\partial}{\partial Y_{b}}\right)^{\beta} \\
\quad \times \int_{0}^{\infty} d \lambda \lambda^{m_{a}-\alpha-m_{b}+\beta-1} \frac{1}{\left(-\lambda P_{a}^{\mp}-\lambda^{-1} P_{b}^{\mp}\right)^{n_{+}+n_{-}+2}} \frac{1}{\left(-\left(\lambda Y_{a}+\frac{1}{\lambda} Y_{b}\right)^{2}\right)^{\frac{d-2}{2}}} .
\end{gathered}
$$

Using the fact that $E_{0} \cdot E_{0}=0$, we can further simplify this to obtain the final expression used in the main text.

\section{E Quicker argument for the vanishing of the modular Hamiltonian term}

We would like to give a quick argument that the second line of (3.12) vanishes. We will do this without passing to the hyperbolic coordinates as in the main text. We will also not make any attempt to explicitly calculate the modular Hamiltonian integral. Rather our argument here will be based on scaling symmetry and the operator product expansion in the CFT. We cut off the integrals over the stress tensor close to the entangling surface by cutting out a tubular region around $\partial A$ of radius $\delta$ and only integrate over the remaining region $U_{\delta}$. Our goal will be to show that this term vanishes as we remove the cutoff $\delta \rightarrow 0$. This cutoff is related to the cutoff in hyperbolic space used in section 3.2 via $\delta=1 / \Lambda$. So recall that the term of interest is

$$
\delta S_{E E}^{(1)}=\frac{1}{2} \int_{U_{\delta}} d^{d} \boldsymbol{x}_{a} \int_{U_{\delta}} d^{d} \boldsymbol{x}_{b} \partial_{\mu} \zeta_{\nu}\left(\boldsymbol{x}_{a}\right) \partial_{\lambda} \zeta_{\sigma}\left(\boldsymbol{x}_{b}\right)\left\langle\widehat{T}^{\mu \nu}\left(\boldsymbol{x}_{a}\right) \widehat{T}^{\lambda \sigma}\left(\boldsymbol{x}_{b}\right) \widehat{H}_{E}\right\rangle .
$$

Integrating by parts on $\boldsymbol{x}_{a}$ and $\boldsymbol{x}_{b}$ and using the diffeomorphism and trace ward identities we can rewrite this as:

$$
\delta S_{E E}^{(1)}=\frac{1}{2} \int_{\partial U_{\delta}} \int_{\partial U_{\delta}} n^{\mu}\left(\boldsymbol{x}_{a}\right) \zeta^{\nu}\left(\boldsymbol{x}_{a}\right) n^{\lambda}\left(\boldsymbol{x}_{b}\right) \zeta^{\sigma}\left(\boldsymbol{x}_{b}\right)\left\langle\widehat{T}^{\mu \nu}\left(\boldsymbol{x}_{a}\right) \widehat{T}^{\lambda \sigma}\left(\boldsymbol{x}_{b}\right) \widehat{H}_{E}\right\rangle+\text { contact terms. }
$$

In appendix $\mathrm{D}$, we have already shown that the contact terms vanish in the limit $\delta=$ $1 / \Lambda \rightarrow 0$, and so we focus here on the remaining term coming from the boundary of the tubular region.

Of course had we not cut off the integral around the tubular region then we would be done - the diffeomorphism Ward identity would leave us with just the contact terms. However we choose to worry about potential divergences around the entangling surface for several reasons. Firstly such terms are generic in entanglement entropy calculations although, since we are calculating a finite quantity (the non-local part of the entanglement 
density), one might expect this to not be an issue. Secondly, when we computed the relative entropy term, an enhancement occured in this region which ruins the naive argument that this term should vanish at least as $\delta^{2}$; here we are checking that such an enhancement does not occur for the modular Hamiltonian term.

At this stage it is convenient to rewrite the boundary term in (E.2) as a bulk integral inside the tubular region $\overline{U_{\delta}}$

$$
\begin{aligned}
\delta S_{E E}^{(1)}= & \frac{1}{2} \int_{\overline{U_{\delta}}} d^{d} \boldsymbol{x}_{a} \int_{\overline{U_{\delta}}} d^{d} \boldsymbol{x}_{b} \int_{0}^{\infty} d x^{1} x^{1} \\
& \times \int d^{d-2} x^{i} \partial_{\lambda} \zeta_{\sigma}\left(\boldsymbol{x}_{a}\right) \partial_{\mu} \zeta_{\nu}\left(\boldsymbol{x}_{a}\right)\left\langle\widehat{T}^{\mu \nu}\left(\boldsymbol{x}_{a}\right) \widehat{T}^{\lambda \sigma}\left(\boldsymbol{x}_{b}\right) \widehat{T}_{00}\left(0, x^{1}, x^{i}\right)\right\rangle
\end{aligned}
$$

where again there is a contact term we have dropped based on the analysis in appendix D. This form is now convenient because we can argue that the leading contribution as $\delta \rightarrow 0$ is:

$$
\begin{aligned}
\delta S_{E E}^{(1)} \stackrel{?}{=} & \frac{\left(\pi \delta^{2}\right)^{2}}{2} \int_{\partial A} \int_{\partial A} \partial_{\mu} \zeta_{\nu}\left(x_{a}^{i}\right) \partial_{\lambda} \zeta_{\sigma}\left(x_{b}^{i}\right) \int_{0}^{\infty} d x^{1} x^{1} \int d^{d-2} x^{i}\left\langle\widehat{T}^{\mu \nu}\left(x_{a}^{i}\right) \widehat{T}^{\lambda \sigma}\left(x_{b}^{i}\right) \widehat{T}^{00}\left(0, x^{1}, x^{i}\right)\right\rangle \\
& +\ldots+\text { contact terms }{ }^{\prime}
\end{aligned}
$$

where we have integrated over the tubular region assuming the relevant integrated threepoint function is a constant over this region. If the term multiplying $\left(\pi \delta^{2}\right)^{2}$ above can be shown to be finite as $\delta \rightarrow 0$ then this assumption is true and further we can argue there are no enhancements from this contribution to the modular Hamiltonian term. Unfortunately this is not quite correct; instead, we will use the OPE of two stress tensors to show that there is at most a logarithmic divergence coming from the $x^{1}$ integral which we should then cut off at small $x^{1} \approx \delta$ close to $\partial A$. This should only lead to a mild enhancement such that the overall scaling of this term is $\delta^{4} \ln \delta$.

The issue comes about for $x^{i} \rightarrow x_{a, b}^{i}$ and $x^{1} \rightarrow 0$ where the stress tensor in the modular Hamiltonian comes close to either one of the two other stress tensor insertions (recall that $x_{a}$ is well separated from $x_{b}$ so we need never consider these two operators colliding) see figure 5 . In this limit we can use the OPE [41]:

$$
\widehat{T}^{00}\left(0, x^{1}, x^{i}\right) \widehat{T}^{\mu \nu}\left(x_{a}^{i}\right) \rightarrow|\sigma|^{-d} A^{00 ; \mu \nu ; \alpha \beta}(\hat{\sigma}) \widehat{T}_{\alpha \beta}\left(x_{a}^{i}\right)+\ldots
$$

where $\sigma=\left(0, x^{1}, x^{i}-x_{a}^{i}\right)$ and $\hat{\sigma}=\sigma /|\sigma|$. The function $A$ depends on several conformally covariant structures with three unfixed theory-dependent parameters. These are the same parameters that appear in the stress tensor three-point function.

Plugging this into the three-point function we find the leading behavior:

$$
\delta S_{E E}^{(1)}=\frac{\left(\pi \delta^{2}\right)^{2}}{2} \int_{\partial A} \int_{\partial A} C^{00 ; \mu \nu ; \alpha \beta} \partial_{\lambda} \zeta_{\sigma}\left(x_{a}^{i}\right) \partial_{\mu} \zeta_{\nu}\left(x_{a}^{i}\right)\left\langle\widehat{T}_{\alpha \beta}\left(x_{a}^{i}\right) \widehat{T}^{\lambda \sigma}\left(x_{b}^{i}\right)\right\rangle+\ldots
$$

where:

$$
C^{00 ; \mu \nu ; \alpha \beta}=\left.\int_{\delta} d x^{1} x^{1} \int d^{d-2} y^{i}|\sigma|^{-d} A^{00 ; \mu \nu ; \alpha \beta}(\hat{\sigma})\right|_{\sigma=\left(0, x^{1}, y^{i}\right)}
$$

This integral is log divergent close to $\sigma=0$ which we cut off by hand at $x^{1} \sim \delta$. We justify this since for $x^{1} \sim \delta$ we cannot replace the integral over the tubular region by an integral 


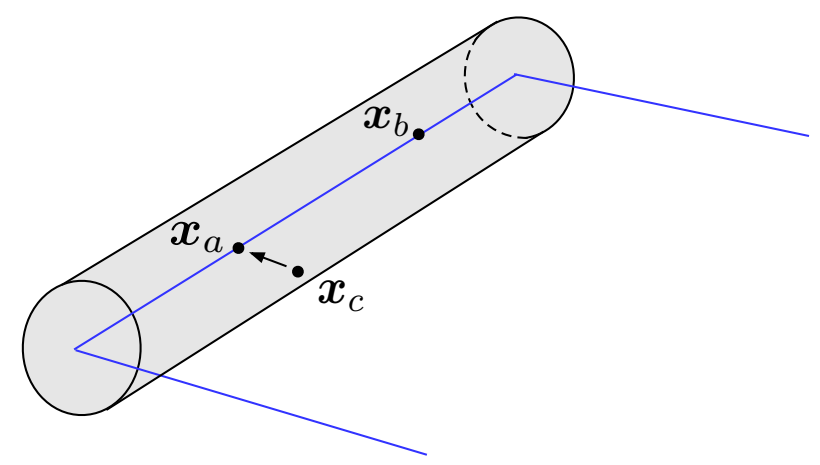

Figure 5. The mild logarithmic enhancement comes from the stress tensor in the modular Hamiltonian coming close to one of the other two stress tensor insertions inside $\overline{U_{\delta}}$.

over $\partial A$. Then we can argue that $C^{00 ; \mu \nu ; \alpha \beta} \sim \alpha \ln \left(\delta /\left|x_{a}^{i}-x_{b}^{j}\right|\right)+\beta$, where the log is cut off at long distances when the OPE expansion breaks down. This argument does not fix $\beta$. It is also possible to show using the same OPE argument that the log is indeed the only enhancement possible for $x^{1} \sim \delta$ and this argument also leads to an explicit and finite expression for $\beta$. We leave this as an exercise to the reader.

We note that the results of this appendix suggest that this contribution to the entanglement density scales as $\delta^{4} \ln \delta$, however in the main text we gave an argument that this term should vanish at least as $\delta^{2}=\Lambda^{-2}$ as suggested in (3.72). For consistency with the results in this appendix the $\mathcal{O}\left(\Lambda^{-2}\right)$ term in (3.72) should actually vanish after we integrate that term over $\tau_{a}$ and $\tau_{b}$ in (3.52) and the leading term should come in at $\mathcal{O}\left(\Lambda^{-4}\right)$ with a possible logarithmic enhancement from the $\lambda$ integral in (3.70). We have checked that this is indeed the case.

Open Access. This article is distributed under the terms of the Creative Commons Attribution License (CC-BY 4.0), which permits any use, distribution and reproduction in any medium, provided the original author(s) and source are credited.

\section{References}

[1] P. Calabrese and J.L. Cardy, Entanglement entropy and quantum field theory, J. Stat. Mech. 0406 (2004) P06002 [hep-th/0405152] [INSPIRE].

[2] P. Calabrese and J. Cardy, Entanglement entropy and conformal field theory, J. Phys. A 42 (2009) 504005 [arXiv: 0905 .4013] [INSPIRE].

[3] H. Casini and M. Huerta, Entanglement entropy in free quantum field theory, J. Phys. A 42 (2009) 504007 [arXiv: 0905.2562] [inSPIRE].

[4] H. Casini, M. Huerta and R.C. Myers, Towards a derivation of holographic entanglement entropy, JHEP 05 (2011) 036 [arXiv:1102.0440] [INSPIRE].

[5] T. Faulkner, The Entanglement Renyi Entropies of Disjoint Intervals in AdS/CFT, arXiv: 1303.7221 [INSPIRE].

[6] T. Faulkner, Bulk Emergence and the RG Flow of Entanglement Entropy, JHEP 05 (2015) 033 [arXiv: 1412.5648] [INSPIRE]. 
[7] A. Kitaev and J. Preskill, Topological entanglement entropy, Phys. Rev. Lett. 96 (2006) 110404 [hep-th/0510092] [INSPIRE].

[8] M. Levin and X.-G. Wen, Detecting topological order in a ground state wave function, Phys. Rev. Lett. 96 (2006) 110405.

[9] S. Dong, E. Fradkin, R.G. Leigh and S. Nowling, Topological Entanglement Entropy in Chern-Simons Theories and Quantum Hall Fluids, JHEP 05 (2008) 016 [arXiv:0802.3231] [INSPIRE].

[10] H. Li and F. Haldane, Entanglement Spectrum as a Generalization of Entanglement Entropy: Identification of Topological Order in Non-Abelian Fractional Quantum Hall Effect States, Phys. Rev. Lett. 101 (2008) 010504 [INSPIRE].

[11] S. Ryu and T. Takayanagi, Holographic derivation of entanglement entropy from AdS/CFT, Phys. Rev. Lett. 96 (2006) 181602 [hep-th/0603001] [INSPIRE].

[12] S. Ryu and T. Takayanagi, Aspects of Holographic Entanglement Entropy, JHEP 08 (2006) 045 [hep-th/0605073] [INSPIRE].

[13] V.E. Hubeny, M. Rangamani and T. Takayanagi, A Covariant holographic entanglement entropy proposal, JHEP 07 (2007) 062 [arXiv:0705.0016] [INSPIRE].

[14] M. Van Raamsdonk, Building up spacetime with quantum entanglement, Gen. Rel. Grav. 42 (2010) 2323 [arXiv: 1005.3035] [INSPIRE].

[15] T. Faulkner, M. Guica, T. Hartman, R.C. Myers and M. Van Raamsdonk, Gravitation from Entanglement in Holographic CFTs, JHEP 03 (2014) 051 [arXiv:1312.7856] [INSPIRE].

[16] M. Nozaki, T. Numasawa and T. Takayanagi, Holographic Local Quenches and Entanglement Density, JHEP 05 (2013) 080 [arXiv: 1302.5703] [INSPIRE].

[17] M. Nozaki, T. Numasawa, A. Prudenziati and T. Takayanagi, Dynamics of Entanglement Entropy from Einstein Equation, Phys. Rev. D 88 (2013) 026012 [arXiv:1304.7100] [INSPIRE].

[18] J. Bhattacharya, V.E. Hubeny, M. Rangamani and T. Takayanagi, Entanglement density and gravitational thermodynamics, Phys. Rev. D 91 (2015) 106009 [arXiv:1412.5472] [INSPIRE].

[19] R. Bousso, Z. Fisher, S. Leichenauer and A.C. Wall, Quantum focusing conjecture, Phys. Rev. D 93 (2016) 064044 [arXiv: 1506.02669] [INSPIRE].

[20] B. Czech, L. Lamprou, S. McCandlish and J. Sully, Integral Geometry and Holography, JHEP 10 (2015) 175 [arXiv: 1505.05515] [INSPIRE].

[21] V. Rosenhaus and M. Smolkin, Entanglement Entropy: A Perturbative Calculation, JHEP 12 (2014) 179 [arXiv: 1403.3733] [INSPIRE].

[22] V. Rosenhaus and M. Smolkin, Entanglement Entropy for Relevant and Geometric Perturbations, JHEP 02 (2015) 015 [arXiv:1410.6530] [InSPIRE].

[23] A. Allais and M. Mezei, Some results on the shape dependence of entanglement and Rnyi entropies, Phys. Rev. D 91 (2015) 046002 [arXiv:1407.7249] [INSPIRE].

[24] P. Bueno, R.C. Myers and W. Witczak-Krempa, Universality of corner entanglement in conformal field theories, Phys. Rev. Lett. 115 (2015) 021602 [arXiv:1505. 04804] [INSPIRE].

[25] P. Bueno and R.C. Myers, Corner contributions to holographic entanglement entropy, JHEP 08 (2015) 068 [arXiv: 1505.07842] [INSPIRE]. 
[26] M. Mezei, Entanglement entropy across a deformed sphere, Phys. Rev. D 91 (2015) 045038 [arXiv: 1411.7011] [INSPIRE].

[27] P. Bueno and R.C. Myers, Universal entanglement for higher dimensional cones, JHEP 12 (2015) 168 [arXiv: 1508.00587] [INSPIRE].

[28] P. Bueno, R.C. Myers and W. Witczak-Krempa, Universal corner entanglement from twist operators, JHEP 09 (2015) 091 [arXiv: 1507.06997] [INSPIRE].

[29] R.-X. Miao, A holographic proof of the universality of corner entanglement for CFTs, JHEP 10 (2015) 038 [arXiv: 1507.06283] [INSPIRE].

[30] P. Bueno and W. Witczak-Krempa, Bounds on corner entanglement in quantum critical states, Phys. Rev. B 93 (2016) 045131 [arXiv:1511.04077] [INSPIRE].

[31] I.R. Klebanov, T. Nishioka, S.S. Pufu and B.R. Safdi, On Shape Dependence and RG Flow of Entanglement Entropy, JHEP 07 (2012) 001 [arXiv:1204.4160] [INSPIRE].

[32] X. Huang, L.-Y. Hung and F.-L. Lin, OPE of the stress tensors and surface operators, JHEP 06 (2015) 087 [arXiv: 1502.02487] [INSPIRE].

[33] D. Carmi, On the Shape Dependence of Entanglement Entropy, JHEP 12 (2015) 043 [arXiv: 1506.07528] [INSPIRE].

[34] H. Elvang and M. Hadjiantonis, Exact results for corner contributions to the entanglement entropy and Rnyi entropies of free bosons and fermions in 3d, Phys. Lett. B 749 (2015) 383 [arXiv: 1506.06729] [INSPIRE].

[35] P. Fonda, D. Seminara and E. Tonni, On shape dependence of holographic entanglement entropy in $A d S_{4} / C F T_{3}, J H E P 12$ (2015) 037 [arXiv:1510.03664] [INSPIRE].

[36] J.J. Bisognano and E.H. Wichmann, On the Duality Condition for Quantum Fields, J. Math. Phys. 17 (1976) 303 [INSPIRE].

[37] S. Banerjee, Wess-Zumino Consistency Condition for Entanglement Entropy, Phys. Rev. Lett. 109 (2012) 010402 [arXiv:1109.5672] [INSPIRE].

[38] H. Casini, M. Huerta, R.C. Myers and A. Yale, Mutual information and the F-theorem, JHEP 10 (2015) 003 [arXiv: 1506.06195] [INSPIRE].

[39] H. Liu and M. Mezei, A Refinement of entanglement entropy and the number of degrees of freedom, JHEP 04 (2013) 162 [arXiv:1202.2070] [INSPIRE].

[40] T. Grover, A.M. Turner and A. Vishwanath, Entanglement Entropy of Gapped Phases and Topological Order in Three dimensions, Phys. Rev. B 84 (2011) 195120 [arXiv:1108.4038] [INSPIRE].

[41] H. Osborn and A.C. Petkou, Implications of conformal invariance in field theories for general dimensions, Annals Phys. 231 (1994) 311 [hep-th/9307010] [INSPIRE].

[42] M.S. Costa, J. Penedones, D. Poland and S. Rychkov, Spinning Conformal Correlators, JHEP 11 (2011) 071 [arXiv: 1107.3554] [inSPIRE].

[43] J.M. Maldacena and G.L. Pimentel, On graviton non-Gaussianities during inflation, JHEP 09 (2011) 045 [arXiv: 1104.2846] [INSPIRE].

[44] S. Giombi, S. Prakash and X. Yin, A Note on CFT Correlators in Three Dimensions, JHEP 07 (2013) 105 [arXiv: 1104.4317] [INSPIRE]. 
[45] H.S. Cohl, On a generalization of the generating function for Gegenbauer polynomials, arXiv: 1105.2735.

[46] R. Szmytkowski, Some integrals and series involving the Gegenbauer polynomials and the Legendre functions on the cut $(-1,1)$, arXiv:1107.2680.

[47] I. Heemskerk, J. Penedones, J. Polchinski and J. Sully, Holography from Conformal Field Theory, JHEP 10 (2009) 079 [arXiv:0907.0151] [INSPIRE].

[48] T. Hartman, Entanglement Entropy at Large Central Charge, arXiv:1303.6955 [INSPIRE].

[49] A. Hamilton, D.N. Kabat, G. Lifschytz and D.A. Lowe, Holographic representation of local bulk operators, Phys. Rev. D 74 (2006) 066009 [hep-th/0606141] [InSPIRE].

[50] L. Bianchi, M. Meineri, R.C. Myers and M. Smolkin, Rényi Entropy and Conformal Defects, arXiv: 1511.06713 [INSPIRE].

[51] A. Lewkowycz and E. Perlmutter, Universality in the geometric dependence of Renyi entropy, JHEP 01 (2015) 080 [arXiv: 1407.8171] [INSPIRE].

[52] S.N. Solodukhin, Entanglement entropy, conformal invariance and extrinsic geometry, Phys. Lett. B 665 (2008) 305 [arXiv:0802.3117] [INSPIRE]. 